\title{
Aging for interacting diffusion processes
}

\author{
Amir Dembo ${ }^{\mathrm{a}, 1}$, Jean-Dominique Deuschel ${ }^{\mathrm{b}, *, 2}$ \\ a Department of Statistics, Stanford University, Stanford, CA 94305, USA \\ b TU Berlin, Fachbereich Mathematik, Strasse des 17 Juni 136, 10623, Berlin, Germany
}

Received 7 July 2005; received in revised form 7 June 2006; accepted 25 July 2006

Available online 13 December 2006

\begin{abstract}
We study the aging phenomenon for a class of interacting diffusion processes $\left\{X_{t}(i), i \in \mathbb{Z}^{d}\right\}$. In this framework we see the effect of the lattice dimension $d$ on aging, as well as that of the class of test functions $f\left(X_{t}\right)$ considered. We further note the sensitivity of aging to specific details, when degenerate diffusions (such as super random walk, or parabolic Anderson model), are considered. We complement our study of systems on the infinite lattice, with that of their restriction to finite boxes. In the latter setting we consider different regimes in terms of box size scaling with time, as well as the effect that the choice of boundary conditions has on aging. The key tool for our analysis is the random walk representation for such diffusions.
\end{abstract}

() 2006 Elsevier Masson SAS. All rights reserved.

\section{Résumé}

Nous considérons le phénomène du vieillissement pour une classe de diffusions en interaction. Dans ce cadre l'effet de la dimension du réseau ainsi que le type des fonctions test sont mis en évidence. Nous notons aussi l'influence de certains paramètres tels que la dégénérescence du coefficient de diffusion, par exemple pour le «super randow walk» ou le modèle d'Anderson parabolique. Nous considérons aussi des systèmes restreints à des boîtes finies. Dans ce cas, la taille de la boîte ainsi que les conditions au bord ont un effet sur le vieillissement. L'outil clef pour notre analyse est la représentation en marche aléatoire.

๑) 2006 Elsevier Masson SAS. All rights reserved.

MSC: primary $60 \mathrm{~K} 35,82 \mathrm{C} 22$; secondary $60 \mathrm{H} 10$

Keywords: Interacting diffusion processes; Aging; Ginzburg-Landau model; Random walk representation

\section{Introduction}

The object of this work is to study out of equilibrium behavior for a certain class of time homogeneous interacting diffusion processes $\left\{X_{t}(i), i \in \mathbb{Z}^{d}\right\}$ on the infinite $d$-dimensional lattice, and for their restriction to finite boxes $i \in B_{N}$. Such diffusion processes appear in many instances. In the context of statistical mechanics, $X_{t}: i \in \mathbb{Z}^{d} \rightarrow X_{t}(i) \in \mathbb{R}$

\footnotetext{
* Corresponding author.

E-mail addresses: amir@stat.stanford.edu (A. Dembo), deuschel@math.tu-berlin.de (J.-D. Deuschel).

1 Research partially supported by NSF grant \#DMS-0406042 and \#DMS-FRG-0244323.

2 Research partially supported by DFG grant \#663/2-3.
} 
also called Ginzburg-Landau model represents the height of a phase separation, cf. [15] and [14]. In the context biological models, $X_{t}$ represent the intensity of a population at site $i$ and time $t \geqslant 0$, cf. [17].

We focus on the phenomenon of aging, which is of much recent interest in the study of out of equilibrium stochastic systems. This topic originated in physics, when experiments in glassy materials demonstrate that "older" systems relax in a slower manner than "younger" ones (cf. [22,12], where the age of a system is the time it spent in the current phase, e.g. at current temperature in a cooling experiment). The same effect has since been found in many statistical physics models, most notably spin glasses, where as the starting time $s$ of observation increases, a much larger time $t$ is required for relaxing the correlation between the configurations of the corresponding dynamical system at times $s$ and $s+t$ to the same prescribed value. For more on aging, see the surveys $[2,4,19]$ in the physics literature and the references therein, or [1] for a review of most mathematical studies of aging completed thus far.

We examine aging here via the time correlations between various test functions, that is,

$$
\operatorname{corr}\left(f\left(X_{S}\right), g\left(X_{S+t}\right)\right) \equiv \frac{\operatorname{cov}\left(f\left(X_{S}\right), g\left(X_{S+t}\right)\right)}{\operatorname{var}\left(f\left(X_{S}\right)\right)^{1 / 2} \operatorname{var}\left(g\left(X_{S+t}\right)\right)^{1 / 2}} .
$$

We thus say that aging takes place in the system (as observed by the test functions $f$ and $g$ ), when the correlation $\operatorname{corr}\left(f\left(X_{s}\right), g\left(X_{s+t}\right)\right)$ decays to zero for some choices of $s, t \rightarrow \infty$ but not for some other choices of $s, t \rightarrow \infty$ (this definition follows $[2,4,19]$ and we note in passing that certain transient Markov processes, such as high-dimensional Brownian motion, shall then exhibit aging).

This framework allows us to examine in Theorem 1.1 the difference in behavior between the recurrent lattice dimensions $d=1,2$ and the transient ones, $d \geqslant 3$, in the context of "local, monotone, approximately linear" test functions, which in Proposition 1.2 we contrast with other classes of test functions, such as local functions of the gradient process, "global" differentiable functions, or highly non-linear and possibly oscillatory, test functions. As we see in Proposition 1.3, the precise degeneracy of the diffusion coefficients can very much alter the way aging is manifested by the system. Further, in the context of dynamics restricted to a large finite box $B_{N}$, we show in Theorem 1.4 that aging is highly sensitive to the scaling of box size $N=N(s, t)$ in terms of time, as well as to the specific type of boundary condition considered. Finally, in Proposition 1.5 we re-examine some of these questions for a variant of the original dynamics, corresponding to the conservation of total volume.

We proceed to present our results, starting with the class of diffusion processes considered here, which are given as the solution of a stochastic differential system (SDS)

$$
X_{t}(i)=x(i)+\int_{0}^{t} b_{i}\left(X_{S}\right) \mathrm{d} s+\int_{0}^{t} \sigma_{i}\left(X_{S}(i)\right) \mathrm{d} W_{s}(i), \quad i \in \mathbb{Z}^{d},
$$

where $\left\{W_{t}(i), i \in \mathbb{Z}^{d}\right\}$ is a family of independent 1-dimensional Wiener processes and the initial configuration is tempered:

$$
x \in E_{r}=\left\{x \in \mathbb{R}^{\mathbb{Z}^{d}}:\|x\|_{r}^{2}=\sum_{i \in \mathbb{Z}^{d}} x(i)^{2} \mathrm{e}^{-r|i|}<\infty\right\} .
$$

We make the following assumptions about the drifts and diffusion coefficients:

Assumption on drift. Let $\mathcal{J}$ be a symmetric, irreducible set of bounded diameter. ${ }^{3}$ Set $\mathcal{J}_{*}=\left\{e=\left(e_{1}, e_{2}\right): e_{2}-e_{1} \in\right.$ $\mathcal{J}\} \subset \mathbb{Z}^{d} \times \mathbb{Z}^{d}$. The drift is of gradient form and depends only on the discrete gradient $\nabla x(e)=x\left(e_{2}\right)-x\left(e_{1}\right), e=$ $\left(e_{1}, e_{2}\right) \in \mathcal{J}_{*}$ :

$$
\begin{aligned}
b_{i}(x) & =-\sum_{j-i \in \mathcal{J}} V_{(i, j)}^{\prime}(x(i)-x(j))=\sum_{e \in \mathcal{J}_{*}: e_{1}=i} V_{e}^{\prime}(\nabla x(e)) \\
& =-\frac{1}{2}\left(\sum_{e \in \mathcal{J}_{*}: e_{2}=i} V_{e}^{\prime}(\nabla x(e))-\sum_{e \in \mathcal{J}_{*}: e_{1}=i} V_{e}^{\prime}(\nabla x(e))\right) \equiv-\operatorname{div}\left(V^{\prime}(\nabla x)\right)(i),
\end{aligned}
$$

\footnotetext{
${ }^{3}$ Symmetric means $i \in \mathcal{J}$ if $-i \in \mathcal{J}$, irreducibility means that for each $i \in \mathbb{Z}^{d}$ we can find $i_{1}, \ldots, i_{k} \in \mathcal{J}$, such that $i=i_{1}+\cdots+i_{k}$ and bounded diameter means $\sup \{|i-j|, i, j \in \mathcal{J}\}<\infty$.
} 
where div is the discrete divergence, $V_{e} \in C^{2}(\mathbb{R})$ are even, strictly convex functions such that for some $0<c_{-} \leqslant$ $c_{+}<\infty$

$$
c_{-} \leqslant V_{e}^{\prime \prime} \leqslant c_{+}, \quad \forall e \in \mathcal{J}_{*} .
$$

A special case deals with linear drifts:

$$
b_{i}(x)=\sum_{j-i \in \mathcal{J}} q(i, j)(x(j)-x(i)),
$$

where $\{q(i, j)\}$ are the jump rates of a symmetric irreducible finite range random walk on $\mathbb{Z}^{d}$ with the uniform ellipticity condition:

$$
c_{-} \leqslant q(i, j) \leqslant c_{+}, \quad \forall(i, j) \in \mathcal{J}_{*} .
$$

A special case is the discrete Laplacian

$$
\Delta x(i)=-\operatorname{div}(\nabla x)(i)=\sum_{j:|j-i|=1}(x(j)-x(i)),
$$

which is the linear drift corresponding to the nearest neighbor simple random walk on $\mathbb{Z}^{d}$.

Assumption on diffusion coefficients. The diffusion coefficients $a_{i}=\sigma_{i}^{2} \in C^{1}(\mathbb{R})$ are uniformly elliptic:

$$
\alpha_{-}^{2} \leqslant a_{i} \leqslant \alpha_{+}^{2}, \quad i \in \mathbb{Z}^{d},
$$

for some $0<\alpha_{-} \leqslant \alpha_{+}<\infty$. Here again, a special case deals with constant (independent of $x$ ) coefficients:

$$
\alpha_{-} \leqslant \sigma_{i}(x(i))=\alpha_{i} \leqslant \alpha_{+}, \quad i \in \mathbb{Z}^{d} .
$$

In this case, the gradient process $\left\{\nabla X_{t}(e), e \in \mathbb{Z}_{*}^{d}\right\}$ is itself a Markov process.

If both (1.3) and (1.5) hold, we have a linear SDS, thus a Gaussian process, the critical Ornstein-Uhlenbeck process:

$$
X_{t}(i)=x(i)+\int_{0}^{t} \sum_{j-i \in \mathcal{J}} q(i, j)\left(X_{S}(j)-X_{S}(i)\right) \mathrm{d} s+\alpha_{i} W_{t}(i), \quad i \in \mathbb{Z}^{d} .
$$

Indeed, aging is examined in [5] for certain Ornstein-Uhlenbeck processes of the type (1.6) and for linear test functions, in which case explicit Gaussian computations are available.

We shall denote by $F(\cdot) \approx G(\cdot)$ the fact that the function $F(\cdot) / G(\cdot)$ is uniformly bounded and bounded away from zero. In particular, all aging functions are specified via $\approx$ up to universal positive, finite constants, which suffices in order to determine whether aging occurs or not. Further, all aging functions are given by the formulas that are valid without any such constants in the Gaussian case, that is, for linear functions $f$ and $g$ and an Ornstein-Uhlenbeck process $X_{s}(\cdot)$.

Under the above assumption the system (1.1) has for each $x \in E_{r}$ a unique solution $X_{t} \in E_{r}, \forall t \geqslant 0$. We denote by $E_{r}^{+}$the non-negative configurations and by $\mathcal{M}_{1}\left(E_{r}\right)$, respectively $\mathcal{M}_{1}\left(E_{r}^{+}\right)$, the probability distributions concentrated on $E_{r}$, respectively $E_{r}^{+}$.

We introduce for each $p \geqslant 1$ the set of differentiable functions

$$
\mathcal{C}_{p}^{1}\left(E_{r}\right)=\left\{f \in C^{1}\left(E_{r}\right):\|f\|_{p} \equiv\left[\sum_{i \in \mathbb{Z}^{d}}\left\|\partial_{i} f\right\|_{\infty}^{p}\right]^{1 / p}<\infty\right\},
$$

where $\partial_{i} f(x) \equiv \partial f(x) / \partial x(i)$. All our test functions will be from $\mathcal{C}_{2}^{1}\left(E_{r}\right)$, and its subset $\mathcal{C}_{1}^{1}\left(E_{r}\right)$ is called the set of local functions.

The initial distribution $v \in \mathcal{M}_{1}\left(E_{r}\right)$ will be a perturbation of i.i.d. measures. More precisely, we assume that

$$
\operatorname{var}_{v}(f) \leqslant\left\|C_{v}\right\|\|f\|_{2}^{2}, \quad \forall f \in \mathcal{C}_{2}^{1}\left(E_{r}\right) .
$$

Note that (1.7) follows from a covariance inequality of the type

$$
\left|\operatorname{cov}_{\nu}(f, g)\right| \leqslant \sum_{i, j} c_{\nu}(i, j)\left\|\partial_{i} f\right\|_{\infty}\left\|\partial_{j} g\right\|_{\infty}, \quad \forall f, g \in \mathcal{C}_{2}^{1}\left(E_{r}\right),
$$


in case $c_{v}(i, j)=c_{v}(j, i) \geqslant 0$ are such that $\sup _{i} \sum_{k} c_{v}(i, k) \equiv\left\|C_{v}\right\|<\infty$. Also whenever a measure $v$ satisfies the FKG property, then (1.8) holds with $c_{v}(i, k)=\sqrt{3} \operatorname{cov}_{v}(x(i), x(k))$, cf. [20].

Next, let $C^{1, \uparrow}\left(E_{r}\right)$ denote the set of coordinate-wise continuous functions $f: E_{r} \rightarrow \mathbb{R}$, that are differentiable with respect to each coordinate of $x \in E_{r}$ and such that $\partial_{i} f(x) \geqslant 0$ for all $x \in E_{r}$ and $i \in \mathbb{Z}^{d}$. Further, let

$$
\mathcal{C}_{1}^{1, \uparrow}\left(E_{r}\right)=\left\{f \in C^{1, \uparrow}\left(E_{r}\right):\|f\|_{1}<\infty,\left|\partial_{i} f\right|_{\text {inf }} \equiv \inf _{x} \partial_{i} f(x)>0 \text {, for some } i \in \mathbb{Z}^{d}\right\},
$$

be the set of monotone increasing local functions, with respect to which we shall usually examine the aging phenomena.

In this context, our next result provides insight about the relation between aging and the underlying lattice dimension.

Theorem 1.1. Assume either (1.2) and constant diffusion coefficients (1.5), or (1.4) and linear drifts (1.3). Take an initial distribution $v \in \mathcal{M}_{1}\left(E_{r}\right)$ satisfying (1.7). Then, for all $f, g \in \mathcal{C}_{1}^{1, \uparrow}\left(E_{r}\right)$, in case of transient lattice dimensions, $d \geqslant 3$, we have no aging, that is

$$
\lim _{s, t \rightarrow \infty} \operatorname{corr}_{v}\left(f\left(X_{s}\right), g\left(X_{s+t}\right)\right)=0 .
$$

For recurrent lattice dimensions we have aging, where for $d=1$,

$$
\lim _{s, t \rightarrow \infty, t / s=a} \operatorname{corr}_{v}\left(f\left(X_{s}\right), g\left(X_{s+t}\right)\right) \approx \frac{(1+a / 2)^{1 / 2}-(a / 2)^{1 / 2}}{(1+a)^{1 / 4}},
$$

and for $d=2$, we have,

$$
\lim _{s, t \rightarrow \infty, \log t / \log s=a} \operatorname{corr}_{v}\left(f\left(X_{S}\right), g\left(X_{s+t}\right)\right) \approx(1-a)_{+} .
$$

As we next show, the choice of test functions is very important. In particular if we consider local functions of the gradient process $\left\{\nabla X_{t}, t \geqslant 0\right\}$, then no aging takes place. Also exponential and trigonometric functions, produce no aging. However, non-local functions produce aging even in higher dimensions. More precisely, for $d \geqslant q>d / 2$, consider the set of non-local monotone differentiable functions

$$
\mathcal{C}_{2, q}^{1, \uparrow}\left(E_{r}\right)=\left\{f \in C^{1, \uparrow}\left(E_{r}\right): 0<\inf _{i}(1+|i|)^{q}\left|\partial_{i} f\right|_{\text {inf }}, \sup _{i}(1+|i|)^{q}\left\|\partial_{i} f\right\|_{\infty}<\infty\right\} .
$$

A typical example of such a function $f \in L^{2}(v)$ for $v$ satisfying (1.7) is the linear function

$$
f(x)=\sum_{i}(1+|i|)^{-q}\left(x(i)-m_{v}(i)\right), \quad \text { where } m_{v}(i)=E_{v}[x(i)] .
$$

Note that $\mathcal{C}_{2, q}^{1, \uparrow}\left(E_{r}\right)$ are for $d \geqslant q>d / 2$ subsets of $\mathcal{C}_{2}^{1}\left(E_{r}\right)$ that exclude the local monotone functions $\mathcal{C}_{1}^{1, \uparrow}\left(E_{r}\right)$.

Then, we have the following result.

\section{Proposition 1.2.}

(i) Let $d \geqslant 2$ and assume (1.2) and constant diffusion coefficients (1.5). Then, for each $f, g \in \mathcal{C}_{1}^{1}\left(E_{r}\right)$ with $f(x)=$ $\tilde{f}(\nabla x), g(x)=\tilde{g}(\nabla x)$

$$
\lim _{s, t \rightarrow \infty} \operatorname{corr}_{v}\left(\tilde{f}\left(\nabla X_{S}\right), \tilde{g}\left(\nabla X_{t+s}\right)\right)=0 .
$$

(ii) Under the assumptions of Theorem 1.1 , take $d \geqslant 3$ and $f, g \in \mathcal{C}_{2, q}^{1, \uparrow}\left(E_{r}\right)$ with $q=(d+1) / 2$ or $q=(d+2) / 2$. Then, aging takes place and (1.10), respectively (1.11), holds.

(iii) Assume that $X_{0}(i)=0$ for all $i \in \mathbb{Z}^{d}$, both linear drifts (1.3) and constant diffusion coefficients (1.5), i.e. consider the Ornstein-Uhlenbeck process (1.6), then for

$$
f, g \in \mathcal{E}_{\rho} \equiv\left\{\rho\left(\sum_{\alpha=1}^{n} c_{j_{\alpha}} x\left(j_{\alpha}\right)\right), c_{j}>0\right\}
$$

no aging takes place (i.e. (1.9) holds), when either $\rho=\exp (\cdot)$ or $\rho=\cos (\cdot)$, or $\rho=\sin (\cdot)$. 
Example 1 (Ginzburg-Landau model). Here we assume a nearest neighbor interaction:

$$
b_{i}(x)=-\sum_{j:|i-j|=1} V^{\prime}(x(i)-x(j)),
$$

and a constant diffusivity coefficient $\sigma_{i}^{2}=\alpha^{2}$. Introducing the formal Hamiltonian

$$
H(x)=\frac{1}{2} \sum_{i, j:|i-j|=1} V(x(i)-x(j)),
$$

we see that this drift is given in terms of the partial derivatives $b_{j}(x)=-\partial_{j} H(x)$ of the Hamiltonian. The SDS (1.1) is thus the Langevin dynamic associated with the Gibbs measure

$$
\mu(d x)=\frac{1}{Z} \exp \left(-\frac{2}{\alpha^{2}} H(x)\right) \prod_{j \in \mathbb{Z}^{d}} \lambda(d x(j)),
$$

$\lambda$ being the Lebesgue measure on $\mathbb{R}$. Of course, (1.13) is just formal and is well defined only on finite box with fixed boundary condition (cf. (1.21) in the sequel). In fact due to the continuous symmetry, that is $H(x)=H(x+c), \forall c \in \mathbb{R}$, no infinite Gibbs state exists on the whole of $\mathbb{Z}^{d}$ for lower lattice dimensions $d=1,2$, cf. [13].

Note however, that aging is not equivalent to the non-existence of an infinite Gibbs state. For example, the dynamic with repulsion:

$$
X_{t}(i)=x(i)+\int_{0}^{t} b_{i}\left(X_{s}\right) \mathrm{d} s+\ell_{t}(i)+\alpha_{i} W_{t}(i), \quad i \in \mathbb{Z}^{d},
$$

where $\ell_{t}(i)$ is the local time at 0 for $X_{s}(i), s \leqslant t$, is delocalized and thus no infinite Gibbs state exists for any dimension $d \geqslant 1$, cf. [10]. Adapting the proof of Theorem 1.1 for the dynamic (1.14) with drift satisfying (1.2) and initial distribution $v \in \mathcal{M}_{1}\left(E_{r}\right)$ satisfying (1.7), the representation of [11, Theorem 2 and Remark 1] yields upper bounds on covariances that are similar to those we have for the same dynamic without the repulsion term $\ell_{t}(i)$. We consequently deduce that even with the repulsion term, no aging takes place when $d \geqslant 3$, that is, (1.9) then holds for all $f, g \in \mathcal{C}_{1}^{1, \uparrow}\left(E_{r}\right)$.

The aging behavior is quite sensitive to the details of degenerate diffusion coefficients. Our next result illustrates this for three different model examples, each of which is of considerable independent interest.

Proposition 1.3. Consider local monotone functions $f, g \in \mathcal{C}_{1}^{1, \uparrow}\left(E_{r}\right)$.

(i) Let $\left(X_{t}\right)$ be the solution of the SDS

$$
X_{t}(i)=x(i)+\int_{0}^{t} b_{i}\left(X_{S}\right) \mathrm{d} s+\int_{0}^{t} 1_{0}(i) \sigma_{i}\left(X_{s}(i)\right) \mathrm{d} W_{s}(i), \quad i \in \mathbb{Z}^{d},
$$

where the drift, the diffusion coefficients and the initial distribution are as in Theorem 1.1. Then, no aging takes place if $d \geqslant 2$, while in this case (1.11) holds for $d=1$.

(ii) Consider the super random walk, that is, the solution of the SDS

$$
X_{t}(i)=x(i)+\int_{0}^{t}\left(\Delta X_{s}\right)(i) \mathrm{d} s+\int_{0}^{t} \alpha X_{s}^{1 / 2}(i) \mathrm{d} W_{s}(i), \quad i \in \mathbb{Z}^{d},
$$

with $\alpha>0$ and an initial measure $v \in \mathcal{M}_{1}\left(E_{r}^{+}\right)$satisfying (1.7) for which $m_{v}(i)=m$ for all $i \in \mathbb{Z}^{d}$, cf. [17]. Then, (1.9), (1.10) and (1.11) hold for $d \geqslant 3, d=1$ and $d=2$, respectively, just as in Theorem 1.1. 
(iii) Consider the parabolic Anderson model, that is, the solution of the SDS

$$
X_{t}(i)=x(i)+\int_{0}^{t}\left(\Delta X_{S}\right)(i) \mathrm{d} s+\int_{0}^{t} \alpha X_{S}(i) \mathrm{d} W_{s}(i), \quad i \in \mathbb{Z}^{d},
$$

with $\alpha>0$ and initial condition $v \in \mathcal{M}_{1}\left(E_{r}^{+}\right)$composed of i.i.d. coordinates of positive mean and finite variance, cf. [3]. Then, no aging takes place, at all dimensions.

This last result just shows that no aging takes place in the parabolic Anderson model for approximately linear test functions, but of course it does not exclude aging for other test functions. In fact this is best understood in view of non-aging for exponentials of the Ornstein-Uhlenbeck process, cf. part (iii) of Proposition 1.2, since both SDS of $\left\{X_{t}\right\}$ in (1.17) and $\left\{\exp \left(X_{t}\right)\right\}$ for $X_{t}$ of (1.1) are very similar. In fact we expect that aging takes place for $f, g \in \mathcal{E}_{\log (\cdot)}$ in $d=1,2$ for any $\alpha>0$ and in $d \geqslant 3$ for all $\alpha>\alpha_{c}(d)>0$.

We now turn to dynamics on a finite box and study the effect the different boundary conditions have on whether the system exhibits aging or not. To this end, let $B_{N}=[0, N-1]^{d} \cap \mathbb{Z}^{d}$ be the box of size $N$. We will consider a dynamic $X_{t}^{N}(i)$ for $i \in B_{N}$ which is the solution of SDS (1.1) restricted to $B_{N}$ with drift $b_{i}^{N}$ corresponding to different types of boundary conditions, as follows.

Fixed boundary conditions. For a given fixed $y \in E_{r}$,

$$
b_{i}^{N}(x)=-\sum_{j \in(\mathcal{J}+i) \cap B_{N}} V_{(i, j)}^{\prime}(x(i)-x(j))-\sum_{j \in(\mathcal{J}+i) \cap B_{N}^{c}} V_{(i, j)}^{\prime}(x(i)-y(j)) .
$$

Free boundary condition.

$$
b_{i}^{N}(x)=-\sum_{j \in(\mathcal{J}+i) \cap B_{N}} V_{(i, j)}^{\prime}(x(i)-x(j)) .
$$

Periodic boundary condition.

$$
b_{i}^{N}(x)=-\sum_{j \in(\mathcal{J}+i)} V_{(i, j)}^{\prime}(x(i)-x(j \bmod N)) .
$$

We observe in passing that setting all diffusion coefficients to the same finite, positive constant $\alpha$, the drift (1.18) corresponds to a Langevin dynamic associated with the Gibbs measure on $B_{N}$ equipped with the boundary condition $y \in \mathbb{R}^{B_{N}^{c}}$, that is,

$$
\mu_{B_{N}}^{y}(\mathrm{~d} x)=\frac{1}{Z_{N}^{y}} \exp \left(-\frac{2}{\alpha^{2}} H_{N}^{y}(x)\right) \prod_{j \in B_{N}} \lambda(\mathrm{d} x(j)) \prod_{j \notin B_{N}} \delta_{y(j)}(\mathrm{d} x(j)),
$$

where

$$
H_{N}^{y}(x)=\frac{1}{2} \sum_{j, i \in B_{N}: j-i \in \mathcal{J}} V(x(i)-x(j))+\sum_{i \in B_{N}, j \notin B_{N}: j-i \in \mathcal{J}} V(x(i)-y(j)) .
$$

However, no Gibbs distribution exists for the periodic or free boundary conditions in this model.

We will fix a time horizon $s+t$ and consider a solution $\left\{X_{u}^{N}, 0 \leqslant u \leqslant s+t\right\}$ with fixed $N$, allowing also for $N=N(s, t) \rightarrow \infty$ as $s, t \rightarrow \infty$. We next examine the sensitivity of aging with respect to boundary conditions and with respect to the box size scaling. Indeed, for fixed b.c. we find aging only for sufficiently large box size, in which case the dynamics behaves as if it is on the infinite lattice, hence also requiring $d \leqslant 2$. In contrast, even for $d \geqslant 3$ aging is present for free or periodic b.c. if the dynamics can feel the boundary condition, that is, when the box size grows slowly enough with respect to time.

Theorem 1.4. Consider a dynamic on a finite box $B_{N}$ under the same hypothesis as Theorem 1.1. 
(i) For fixed b.c. as in (1.18), the conclusions of Theorem 1.1 apply when $s, t=\mathrm{o}\left(N^{2} / \log N\right)$, whereas when $N(s, t)=\mathrm{o}\left((t / \log t)^{1 / 2}\right)$ no aging takes place, for all $d \geqslant 1$.

(ii) For $d \geqslant 3$ and either free or periodic b.c. as in (1.19) or (1.20), no aging takes place when $s=\mathrm{o}\left(N^{d}\right)$, whereas if $N=\mathrm{o}\left(s^{1 / d}\right)$, then

$$
\lim _{s, t \rightarrow \infty, t / s=a} \operatorname{corr}_{v}\left(f\left(X_{s}\right), g\left(X_{s+t}\right)\right) \approx \frac{1}{\sqrt{a+1}} .
$$

Another interesting variation of the Ginzburg-Landau model, cf. [21], is the dynamic with preserved volume, as follows. We denote by $T_{N}$ the discrete torus $(-N / 2, N / 2]^{d} \cap \mathbb{Z}^{d}$ equipped with periodic bonds $T_{N}^{*}=\left\{e=\left(e_{1}, e_{2}\right)\right.$ : $\left.e_{i} \in T_{N},\left|e_{1}-e_{2} \bmod N\right|=1\right\}$. Recall the definition of the discrete divergence

$$
\operatorname{div} f(i)=\frac{1}{2}\left(\sum_{e \in T_{N}^{*}, e_{2}=i} f(e)-\sum_{e \in T_{N}^{*}, e_{1}=i} f(e)\right)
$$

and the discrete periodic Laplacian $\Delta f(i)=-\operatorname{div}(\nabla f)(i)$. For a family $\left\{W_{t}(e), e \in T_{N}^{*}, t \geqslant 0\right\}$ of independent Brownian motions, we set $\widehat{W}_{t}(i)=\sqrt{2} \operatorname{div}\left(W_{t}\right)(i)$, noting that

$$
\operatorname{cov}\left(\widehat{W}_{s}(i), \widehat{W}_{t}(j)\right)=(t \wedge s)\left(-\Delta 1_{i}\right)(j) .
$$

A direct application of Ito's formula shows that the total volume in the dynamics

$$
X_{t}(i)=x(i)+\int_{0}^{t} \Delta b \cdot\left(X_{s}\right)(i) \mathrm{d} s+\alpha \widehat{W}_{t}(i), \quad i \in T_{N},
$$

remains constant over time. That is,

$$
\operatorname{vol}\left(X_{t}\right) \equiv \sum_{i \in T_{N}} X_{t}(i)=\operatorname{vol}\left(X_{0}\right), \quad \forall t \geqslant 0 .
$$

Further, for each given $v \in \mathbb{R}$, the SDS (1.23) is the Langevin dynamic associated with a micro-canonical Gibbs distribution on $T_{N}$ with fixed $\operatorname{vol}(x)=v$ (cf. [21]). For this dynamics, and for linear drifts, we have the following (aging) behavior.

Proposition 1.5. Consider the SDS

$$
X_{t}(i)=x(i)-\int_{0}^{t}(\Delta)^{2}\left(X_{s}\right)(i) \mathrm{d} s+\alpha \widehat{W}_{t}(i), \quad i \in T_{N} .
$$

Then, for $f(x)=f(x(0))$ and $g(x)=g(x(0))$ with bounded and bounded away from zero derivatives, we have no aging when $N \rightarrow \infty$ and either $d \geqslant 3$, or $d=2$ and $N^{4}=\mathrm{o}(t)$, or $d=1$ and $N^{4} \log N=\mathrm{o}(t)$. On the other hand, if $(t+s) N^{-4}$ is held bounded, then the relation (1.11) holds when $d=2$, while for $d=1$ we have that

$$
\lim _{s, t \rightarrow \infty, t / s=a} \operatorname{corr}_{\nu}\left(f\left(X_{S}\right), g\left(X_{S+t}\right)\right) \approx \frac{(1+a / 2)^{1 / 4}-(a / 2)^{1 / 4}}{(1+a)^{1 / 8}} .
$$

As our proofs rely on the random walk representation for the SDS (1.1), we devote the next section to a short exposition of this approach, with Section 3 containing the proof of all results pertaining to SDS on the infinite lattice, and Section 4 containing our counterpart results for the dynamics on a finite box.

\section{The random walk representation}

We provide here a short overview of the random walk representation, cf. [9] or [8], see also [16,18], on which most of our proofs are based. To this end, we denote by $\left(P_{t}, t \geqslant 0\right)$ the semi-group for the SDS (1.1) and by $L$ its generator. Let

$$
\mathcal{C}_{1}^{2}\left(E_{r}\right)=\left\{f \in C^{2}\left(E_{r}\right): \sum_{i}\left\|\partial_{i} f\right\|_{\infty}+\sum_{i}\left\|\partial_{i}^{2} f\right\|_{\infty}<\infty\right\} .
$$


Acting on $f \in \mathcal{C}_{1}^{2}\left(E_{r}\right)$, we have

$$
L f(x)=\sum_{i}\left(b_{i}(x) \partial_{i} f(x)+\frac{1}{2} a_{i}(x(i)) \partial_{i}^{2} f(x)\right),
$$

where $a_{i}=\sigma_{i}^{2}$ are such that $\sup _{i, y} a_{i}(y)<\infty$. Next let $\Gamma$ be the bilinear form on $\mathcal{C}_{1}^{2}\left(E_{r}\right) \times \mathcal{C}_{1}^{2}\left(E_{r}\right)$,

$$
\begin{aligned}
\Gamma(f, g)(x) & =L(f g)(x)-f(x) L g(x)-g(x) L f(x) \\
& =\sum_{i} a_{i}(x(i)) \partial_{i} f(x) \partial_{i} g(x),
\end{aligned}
$$

with $\Gamma(f, g)$ then defined as $\sum_{i} a_{i}(x(i)) \partial_{i} f(x) \partial_{i} g(x)$ which is finite for all $f, g \in \mathcal{C}_{2}^{1}\left(E_{r}\right)$.

In view of Proposition 1.3 of [8], we have that

$$
\operatorname{cov}_{v}\left(f\left(X_{s}\right), g\left(X_{s+t}\right)\right)=\operatorname{cov}_{v}\left(P_{s} f, P_{s+t} g\right)+\int_{0}^{s} \mathbb{E}\left[\Gamma\left(P_{u} f, P_{u+t} g\right)\left(X_{s-u}\right)\right] \mathrm{d} u,
$$

for any $f, g \in \mathcal{C}_{2}^{1}\left(E_{r}\right)$. It is not hard to show that $\mathcal{C}_{2}^{1}\left(E_{r}\right)$ is invariant under the semi-group $\left(P_{t}, t \geqslant 0\right)$, and by (1.7) it is also a subset of $L^{2}(v)$. Therefore, while the random walk representation and in particular the decomposition formula (2.3) are derived in [8] only for local functions $f, g \in \mathcal{C}_{1}^{1}\left(E_{r}\right)$, one can adapt the proof so it applies for all functions in $\mathcal{C}_{2}^{1}\left(E_{r}\right)$.

Recall (1.7), due to which we just need an estimate for $\partial_{i} P_{t} f$ in order to use (2.3). We consider first the case (1.5), with constant diffusion coefficients $\sigma_{i}$. Let $\bar{X}_{t}=\left(\xi_{t}, X_{t}\right) \in \mathbb{Z}^{d} \times E_{r}$ be the process generated by

$$
\bar{L} f(i, x)=(L f(i, \cdot))(x)+\sum_{j: j-i \in \mathcal{J}} V_{(i, j)}^{\prime \prime}(x(i)-x(j))(f(j, x)-f(i, x)) .
$$

That is, the second coordinate $\left(X_{t}, t \geqslant 0\right)$ is just the original diffusion solution of the SDS (1.1), while for a given realization $\left(X_{t}, t \geqslant 0\right)$, the process $\left(\xi_{t}, t \geqslant 0\right)$ is the time inhomogeneous random walk on $\mathbb{Z}^{d}$ with symmetric jump rates from $i$ to $j$ given by $V_{(i, j)}^{\prime \prime}\left(X_{t}(i)-X_{t}(j)\right)$. The random walk representation gives

$$
\partial_{i} P_{t} f(x)=\sum_{j \in \mathbb{Z}^{d}} \mathbb{E}_{i, x}\left[\partial_{j} f\left(X_{t}\right) 1_{j}\left(\xi_{t}\right)\right]=\sum_{j \in \mathbb{Z}^{d}} \mathbb{E}_{x}\left[\partial_{j} f\left(X_{t}\right) p_{t}^{X}(i, j)\right],
$$

where $X_{0}=x$ and

$$
p_{t}^{X}(i, j)=\mathbb{E}_{i}\left[1_{j}\left(\xi_{t}\right) \mid \mathcal{F}_{t}^{X}\right],
$$

is the conditional probability of the random walk starting at $i$ to be at time $t$ in position $j$ given $\mathcal{F}_{t}^{X} \equiv \sigma\left(X_{s}, 0 \leqslant s \leqslant t\right)$.

Let $p_{t}^{*}(i-j)=\mathbb{P}_{i}\left(\xi_{t}^{*}=j\right)$ denote the transition function of the nearest neighbor simple random walk on $\mathbb{Z}^{d}$. It is well known that $p_{t}^{*}(i-j)$ behaves for large time $t \geqslant|i-j|$ like the Gaussian kernel and for large $|i-j|$ like the Poissonian kernel, that is, we can find constants ${ }^{4} c_{i}$, such that, for any $i, j \in \mathbb{Z}^{d}$ and all $t \geqslant 0$,

$$
\frac{c_{1}}{1+t^{d / 2}} \exp \left(-E\left(c_{2} t,|i-j|\right)\right) \leqslant p_{t}^{*}(i-j) \leqslant \frac{c_{3}}{1+t^{d / 2}} \exp \left(-E\left(c_{4} t,|i-j|\right)\right),
$$

where (cf. Proposition 3.4 [6]),

$$
E(t, r)=\sup _{\lambda}\{r \lambda-t(\cosh \lambda-1)\}=r \cdot \arg \sinh \left(\frac{r}{t}\right)-t\left(\sqrt{1+\frac{r^{2}}{t^{2}}}-1\right) .
$$

Next, set $\bar{p}_{t}(i, j)=\sup _{X} p_{t}^{X}(i, j)$ and $\underline{p}_{t}(i, j)=\inf _{X} p_{t}^{X}(i, j)$. In view of inequality (1.2) of [9] we can estimate $\bar{p}_{t}(i, j)$ and $\underline{p}_{t}(i, j)$ in terms of $p_{t}^{*}(i-j)$. More precisely, we can find constants $c_{i}$ such that for any $t \geqslant 0, i, j \in \mathbb{Z}^{d}$,

$$
c_{1} p_{t c_{2}}^{*}(i-j) \leqslant \underline{p}_{t}(i, j) \leqslant \bar{p}_{t}(i, j) \leqslant c_{3} p_{t c_{4}}^{*}(i-j) .
$$

\footnotetext{
${ }^{4}$ In what follows, $c_{1}, c_{2}, c_{3}, c_{4}$ are positive constant, which do not depend the time $t$, but may differ from line to line.
} 
In case of linear drift but non-constant diffusion coefficients, consider the process $\bar{X}_{t}=\left(\hat{\xi}_{t}, \widehat{X}_{t}\right)$ generated by

$$
\bar{L} f(i, x)=(L f(i, \cdot))(x)+\frac{1}{2} a_{i}^{\prime}(x(i)) \partial_{i} f(i, x)+\sum_{j \in(\mathcal{J}+i)} q(i, j)(f(j, x)-f(i, x)) .
$$

Thus $\left(\hat{\xi}_{t}, t \geqslant 0\right)$ is a random walk with symmetric, time homogeneous jump rate, while for given $\left(\hat{\xi}_{t}, t \geqslant 0\right)$, the diffusion process $\left(\widehat{X}_{t}, t \geqslant 0\right)$ is the solution of the SDS

$$
\mathrm{d} \widehat{X}_{t}(i)=\left(b_{i}\left(\widehat{X}_{t}\right)+\frac{1}{2} 1_{i}\left(\hat{\xi}_{t}\right) a_{i}^{\prime}\left(\widehat{X}_{t}(i)\right)\right) \mathrm{d} t+\sigma_{i}\left(\widehat{X}_{t}(i)\right) \mathrm{d} W_{t}(i), \quad i \in \mathbb{Z}^{d} .
$$

The random walk representation gives in this case (cf. Theorem 1.1 of [8]),

$$
\partial_{i} P_{t} f(x)=\sum_{j \in \mathbb{Z}^{d}} \mathbb{E}_{i, x}\left[\partial_{j} f\left(\widehat{X}_{t}\right) 1_{j}\left(\hat{\xi}_{t}\right)\right] .
$$

Let $\hat{p}_{t}(i, j)=\mathbb{P}_{i}\left(\hat{\xi}_{t}=j\right)$ denote the transition function of the random walk $\hat{\xi}_{t}$ on $\mathbb{Z}^{d}$, noting that the estimate (2.6) then applies also for $\hat{p}_{t}(i, j)$ (again, by inequality (1.2) of [9]).

\section{Aging for interacting diffusions on $\mathbb{Z}^{d}$}

Equipped with the random walk representation, we next prove our main result, Theorem 1.1, dealing with aging for local monotone test functions.

Proof of Theorem 1.1. Take $f \in \mathcal{C}_{1}^{1}\left(E_{r}\right)$. Then, using either (2.4) or (2.7), in combination with (2.6), we get that

$$
\left\|\partial_{i} P_{t} f\right\|_{\infty} \leqslant \sum_{l} c_{3} p_{t c_{4}}^{*}(i-l)\left\|\partial_{l} f\right\|_{\infty}
$$

In particular, for $f \in \mathcal{C}_{1}^{1}\left(E_{r}\right)$ and $v \in \mathcal{M}_{1}\left(E_{r}\right)$ satisfying (1.7), we can find a constant $c_{1}(\nu, f)<\infty$ such that by (2.5) and (3.1),

$$
\begin{aligned}
\operatorname{var}_{v}\left(P_{s} f\right) & \leqslant\left\|C_{v}\right\| \sum_{i, j, k} c_{3}^{2} p_{s c_{4}}^{*}(i-j)\left\|\partial_{j} f\right\|_{\infty} p_{s c_{4}}^{*}(i-k)\left\|\partial_{k} f\right\|_{\infty} \\
& =\left\|C_{v}\right\| c_{3}^{2} \sum_{j, k} p_{2 s c_{4}}^{*}(k-j)\left\|\partial_{j} f\right\|_{\infty}\left\|\partial_{k} f\right\|_{\infty} \\
& \leqslant c_{3}^{2}\|f\|_{1}^{2}\left\|C_{v}\right\| \max _{i}\left\{p_{2 s c_{4}}^{*}(i)\right\} \leqslant c_{1}(v, f)(s+1)^{-d / 2} .
\end{aligned}
$$

Applying the same bound for $\operatorname{var}_{v}\left(P_{S+t} g\right)$ we get that

$$
\begin{aligned}
\left|\operatorname{cov}_{v}\left(P_{s} f, P_{s+t} g\right)\right| & \leqslant \operatorname{var}_{\nu}\left(P_{s} f\right)^{1 / 2} \operatorname{var}_{v}\left(P_{s+t} g\right)^{1 / 2} \\
& \leqslant \sqrt{c_{1}(\nu, f) c_{1}(v, g)}(s+1)^{-d / 4}(s+t+1)^{-d / 4} .
\end{aligned}
$$

For $\Gamma$ of (2.2) and with $a_{i}(x(i))$ bounded, by (3.1) and (2.5) we can similarly find $c_{2}(f, g)<\infty$ such that

$$
\Gamma\left(P_{s} f, P_{s+t} g\right) \leqslant c_{2}(f, g)(2 s+t+1)^{-d / 2} .
$$

Using again (2.4) or (2.7), in combination with (2.6), we get that

$$
\left|\partial_{i} P_{t} f\right|_{\text {inf }} \geqslant c_{1} \sum_{j} p_{t c_{2}}^{*}(i-j)\left|\partial_{j} f\right|_{\text {inf. }}
$$

Recall that if $f, g \in \mathcal{C}_{1}^{1, \uparrow}\left(E_{r}\right)$ then $\left|\partial_{l} f\right|_{\text {inf }}>0$ and $\left|\partial_{m} g\right|_{\text {inf }}>0$ for some $l, m \in \mathbb{Z}^{d}$, hence by uniform ellipticity and (2.5) we have the lower bound 


$$
\begin{aligned}
\Gamma\left(P_{s} f, P_{s+t} g\right) & \geqslant\left(c_{1} \alpha_{-}\right)^{2} \sum_{i, j, k} p_{s c_{2}}^{*}(i-j) p_{(s+t) c_{2}}^{*}(i-k)\left|\partial_{j} f\right|_{\text {inf }}\left|\partial_{k} g\right|_{\text {inf }} \\
& \geqslant\left(c_{1} \alpha_{-}\right)^{2} p_{(2 s+t) c_{2}}^{*}(l-m)\left|\partial_{l} f\right|_{\text {inf }}\left|\partial_{m} g\right|_{\text {inf }} \\
& \geqslant c_{3}(f, g)(2 s+t+1)^{-d / 2},
\end{aligned}
$$

for some $c_{3}(f, g)>0$. Thus, in view of (2.3), we see that

$$
\operatorname{var}_{v}\left(f\left(X_{s}\right)\right) \approx \begin{cases}(s+1)^{1 / 2}, & d=1 \\ \log (s+1), & d=2 \\ 1, & d \geqslant 3\end{cases}
$$

with a similar expression for $\operatorname{var}_{v}\left(g\left(X_{s+t}\right)\right)$, while for some $s_{0}<\infty$ and all $s \geqslant s_{0}, t \geqslant 0$,

$$
\operatorname{cov}_{v}\left(f\left(X_{s}\right), g\left(X_{s+t}\right)\right) \approx \begin{cases}(2 s+t+1)^{1 / 2}-(t+1)^{1 / 2}, & d=1, \\ \log (2 s+t+1)-\log (t+1), & d=2,\end{cases}
$$

whereas for $d \geqslant 3$ and some finite $c_{4}=c_{4}(\nu, f, g)$,

$$
\left|\operatorname{cov}_{v}\left(f\left(X_{s}\right), g\left(X_{s+t}\right)\right)\right| \leqslant c_{4}\left[(s+t+1)^{-d / 4}+(t+1)^{-d / 2+1}-(2 s+t+1)^{-d / 2+1}\right] .
$$

This of course implies the stated results.

Continuing with a similar type of arguments, we next prove Proposition 1.2, about aging as observed by different types of test functions.

Proof of Proposition 1.2. (i) Take $f(x)=\tilde{f}(\nabla x)$, noting that $\partial_{i} f(x)=2 \operatorname{div}(\partial \tilde{f}(\nabla x))(i)$, so by (2.4) and summation by parts,

$$
\partial_{i}\left(P_{t} f\right)(x)=\mathbb{E}_{x}\left[\sum_{j} \partial_{j} f\left(X_{t}\right) p_{t}^{X}(i, j)\right]=\mathbb{E}_{x}\left[\sum_{e} \partial_{\nabla x(e)} \tilde{f}\left(\nabla X_{t}\right) \nabla p_{t}^{X}(i, \cdot)(e)\right] .
$$

Next we have the Nash type inequality, whereby we can find $\epsilon>0$, such that

$$
\left|\nabla p_{t}^{X}(i, \cdot)(e)\right| \leqslant c_{1}(1 \vee t)^{-\epsilon} p_{c_{2} t}^{*}\left(i-e_{1}\right)
$$

cf. inequality (1.3) of [9]. So, we have the bound of (3.1) with the additional factor $t^{-\epsilon}$, from which we proceed as before to show the absence of aging when $d \geqslant 2$.

(ii) Fixing $v \in \mathcal{M}_{1}\left(E_{r}\right)$ for which (1.7) holds, let

$$
h_{\alpha}(x)=\sum_{k}(1+|k|)^{-q_{\alpha}}\left(x(k)-m_{v}(k)\right), \quad \alpha=1,2
$$

where $q_{\alpha}=(d+\alpha) / 2$ and $m_{v}(\cdot)$ is per (1.12). We first study the aging properties of $h_{\alpha} \in \mathcal{C}_{2, q_{\alpha}}^{1, \uparrow}\left(E_{r}\right)$, for which we use the Fourier representation for the simple random walk. To this end, letting

$$
\hat{a}(\theta)=\sum_{|k|=1}\left(1-\mathrm{e}^{\mathrm{i} k \cdot \theta}\right)=2 \sum_{l=1}^{d}\left(1-\cos \theta_{l}\right),
$$

for $\theta=\left(\theta_{1}, \ldots, \theta_{d}\right)$, we have that

$$
{\widehat{p^{*}}}_{t}(\theta)=\sum_{k} p_{t}^{*}(k) \mathrm{e}^{\mathrm{i} k \cdot \theta}=\mathrm{e}^{-t \hat{a}(\theta)} \text {. }
$$

Define

$$
\widehat{\partial h_{\alpha}}(\theta)=\sum_{k}(1+|k|)^{-q_{\alpha}} \mathrm{e}^{\mathrm{i} k \cdot \theta},
$$

noting that for $c_{5}=\left(c_{3} \alpha_{+}\right)^{2}<\infty$, we get by (2.6) along the lines of proof of Theorem 1.1 that, 


$$
\begin{aligned}
\Gamma\left(P_{s} h_{\alpha}, P_{s+t} h_{\alpha}\right) & \leqslant c_{5} \sum_{j, k}\left\|\partial_{j} h_{\alpha}\right\|_{\infty} p_{(2 s+t) c_{4}}^{*}(j-k)\left\|\partial_{k} h_{\alpha}\right\|_{\infty} \\
& =c_{5} \sum_{j, k}(1+|j|)^{-q_{\alpha}}(1+|k|)^{-q_{\alpha}} p_{(2 s+t) c_{4}}^{*}(j-k) \\
& =\frac{c_{5}}{(2 \pi)^{d}} \int_{[-\pi, \pi]^{d}}\left|\widehat{\partial h_{\alpha}}(\theta)\right|^{2} \exp \left(-c_{4}(2 s+t) \hat{a}(\theta)\right) \mathrm{d} \theta,
\end{aligned}
$$

with the last equality due to Plancherel's identity. Further, with $\partial_{j} h_{\alpha}$ independent of $x$, the converse inequality

$$
\Gamma\left(P_{s} h_{\alpha}, P_{s+t} h_{\alpha}\right) \geqslant \frac{c_{6}}{(2 \pi)^{d}} \int_{[-\pi, \pi]^{d}}\left|\widehat{\partial h_{\alpha}}(\theta)\right|^{2} \exp \left(-c_{2}(2 s+t) \hat{a}(\theta)\right) \mathrm{d} \theta,
$$

also holds for $c_{6}=\left(c_{1} \alpha_{-}\right)^{2}>0$ and $c_{2}>0$ of (2.6). Note that for some finite $c_{+}$and positive $c_{-}$,

$$
c_{-}|\theta|^{2} \leqslant \hat{a}(\theta) \leqslant c_{+}|\theta|^{2}, \quad \theta \in[-\pi, \pi]^{d},
$$

and $\left|\widehat{\partial h_{\alpha}}(\theta)\right|^{2} \approx|\theta|^{-2 d+2 q_{\alpha}}$ for $\theta \rightarrow 0$ (cf. Theorem 1.9 of [7]). Consequently, it is not hard to check that as a function of both $x$ and $s, t \geqslant 0$,

$$
\Gamma\left(P_{s} h_{\alpha}, P_{s+t} h_{\alpha}\right) \approx \begin{cases}(2 s+t+1)^{-1 / 2}, & q=q_{1}, \\ (2 s+t+1)^{-1}, & q=q_{2} .\end{cases}
$$

The same argument also provides the bound

$$
\begin{aligned}
\varphi(s) & \equiv \sum_{i}\left[\sum_{k}(1+|k|)^{-q_{\alpha}} p_{s c_{4}}^{*}(i-k)\right]^{2} \\
& =\sum_{j, k}(1+|j|)^{-q_{\alpha}}(1+|k|)^{-q_{\alpha}} p_{2 s c_{4}}^{*}(j-k) \leqslant c_{0}(2 s+1)^{-\alpha / 2},
\end{aligned}
$$

for some $c_{0}=c_{0}\left(c_{4}, d, \alpha\right)<\infty$ and all $s \geqslant 0$.

Turning to the general case of $f, g \in \mathcal{C}_{2, q}^{1, \uparrow}\left(E_{r}\right)$, we can find constants $0<c_{1}<c_{2}<\infty$ such that for all $k \in \mathbb{Z}^{d}$,

$$
c_{1}(1+|k|)^{-q} \leqslant\left|\partial_{k} f\right|_{\text {inf }} \leqslant\left\|\partial_{k} f\right\|_{\infty} \leqslant c_{2}(1+|k|)^{-q} .
$$

Using the random walk representation, we thus see from (2.4) and (2.7) that for $q=q_{\alpha}$, all $k \in \mathbb{Z}^{d}, x \in E_{r}$ and $t \geqslant 0$,

$$
c_{1} \partial_{k} P_{t} h_{\alpha}(x) \leqslant \partial_{k} P_{t} f(x) \leqslant c_{2} \partial_{k} P_{t} h_{\alpha}(x) .
$$

The same applies for $g \in \mathcal{C}_{2, q_{\alpha}}^{1, \uparrow}\left(E_{r}\right)$. Hence, by (2.2) and uniform ellipticity it follows that also,

$$
\Gamma\left(P_{s} f, P_{s+t} g\right) \approx \begin{cases}(2 s+t+1)^{-1 / 2}, & q=q_{1}, \\ (2 s+t+1)^{-1}, & q=q_{2} .\end{cases}
$$

Further, for $v \in \mathcal{M}_{1}\left(E_{r}\right)$ that satisfies (1.7) and any $f, g \in \mathcal{C}_{2, q_{\alpha}}^{1, \uparrow}\left(E_{r}\right)$, we get in view of (3.6) that for some finite $c_{1}(\nu, f, g)$ and $c_{2}(\nu, f, g)$,

$$
\begin{aligned}
\left|\operatorname{cov}_{v}\left(P_{s} f, P_{s+t} g\right)\right| & \leqslant \operatorname{var}_{\nu}\left(P_{s} f\right)^{1 / 2} \operatorname{var}_{\nu}\left(P_{s+t} g\right)^{1 / 2} \\
& \leqslant c_{1} \varphi(s)^{1 / 2} \varphi(s+t)^{1 / 2} \leqslant c_{2}(2 s+1)^{-\alpha / 4}(2(s+t)+1)^{-\alpha / 4} .
\end{aligned}
$$

Since $\operatorname{cov}_{v}\left(P_{s} f, P_{s+t} g\right) \rightarrow 0$ for $s \rightarrow \infty$, uniformly in $t$, we proceed to get the stated aging results from (3.7) just as we did at the end of the proof of Theorem 1.1.

(iii) Considering the Ornstein-Uhlenbeck process starting at deterministic $X_{0}(i)=0$ we take $f(x)=\rho\left(\ell_{1}(x)\right) \in$ $\mathcal{E}_{\rho}$ and $g(x)=\rho\left(\ell_{2}(x)\right) \in \mathcal{E}_{\rho}$ for monotone local functions $\ell_{1}(x)$ and $\ell_{2}(x)$ of the form $\sum_{\alpha=1}^{n} c_{j_{\alpha}} x\left(j_{\alpha}\right)$, with $c_{j_{\alpha}}>0$. Since $\ell_{1}\left(X_{S}\right)$ and $\ell_{2}\left(X_{s+t}\right)$ are then jointly Gaussian and of zero mean, we can compute explicitly the correlations for such $f$ and $g$ when either $\rho=\exp$, or $\rho=\cos$, or $\rho=\sin$, via the following Gaussian identities. 
Lemma 3.1. Let $X, Y$ be jointly Gaussian. Then,

$$
\operatorname{corr}\left(\mathrm{e}^{X}, \mathrm{e}^{Y}\right)=\frac{\exp (\operatorname{cov}(X, Y))-1}{(\exp (\operatorname{var}(X))-1)^{1 / 2}(\exp (\operatorname{var}(Y))-1)^{1 / 2}} .
$$

Further, if both $\mathbb{E}[X]=\mathbb{E}[Y]=0$, then

$$
\operatorname{corr}(\cos (X), \cos (Y))=\frac{\cosh (\operatorname{cov}(X, Y))-1}{(\cosh (\operatorname{var}(X))-1)^{1 / 2}(\cosh (\operatorname{var}(Y))-1)^{1 / 2}},
$$

and

$$
\operatorname{corr}(\sin (X), \sin (Y))=\frac{\sinh (\operatorname{cov}(X, Y))}{(\sinh (\operatorname{var}(X)))^{1 / 2}(\sinh (\operatorname{var}(Y)))^{1 / 2}} .
$$

Proof. Let $\bar{X}=X-\mathbb{E}[X], \bar{Y}=Y-\mathbb{E}[Y]$, then $\operatorname{corr}\left(\mathrm{e}^{X}, \mathrm{e}^{Y}\right)=\operatorname{corr}\left(\mathrm{e}^{\bar{X}}, \mathrm{e}^{\bar{Y}}\right)$, with

$$
\mathbb{E}\left[\mathrm{e}^{\bar{X}+\bar{Y}}\right]=\exp \left(\frac{1}{2} \operatorname{var}(X+Y)\right)=\mathbb{E}\left[\mathrm{e}^{\bar{X}}\right] \mathbb{E}\left[\mathrm{e}^{\bar{Y}}\right] \exp (\operatorname{cov}(X, Y)),
$$

which implies (3.8). Next using the fact that $\mathbb{E}[X]=\mathbb{E}[Y]=0$ we have

$$
\mathbb{E}[\cos (X)]=\mathbb{E}\left[\mathrm{e}^{\mathrm{i} X}\right]=\mathrm{e}^{-\frac{1}{2} \operatorname{var}(X)}
$$

and

$$
\begin{aligned}
\mathbb{E}[\cos (X) \cos (Y)] & =\frac{1}{2} \mathbb{E}\left[\mathrm{e}^{\mathrm{i}(X+Y)}+\mathrm{e}^{\mathrm{i}(X-Y)}\right] \\
& =\frac{1}{2}\left(\exp \left(-\frac{1}{2} \operatorname{var}(X+Y)\right)+\exp \left(-\frac{1}{2} \operatorname{var}(X-Y)\right)\right) \\
& =\mathbb{E}\left[\mathrm{e}^{\mathrm{i} X}\right] \mathbb{E}\left[\mathrm{e}^{\mathrm{i} Y}\right] \cosh (\operatorname{cov}(X, Y)),
\end{aligned}
$$

from which we deduce (3.9), whereas by symmetry $\mathbb{E}[\sin (X)]=\mathbb{E}[\sin (Y)]=0$ and

$$
\begin{aligned}
\mathbb{E}[\sin (X) \sin (Y)] & =-\frac{1}{2} \mathbb{E}\left[\mathrm{e}^{\mathrm{i}(X+Y)}-\mathrm{e}^{\mathrm{i}(X-Y)}\right] \\
& =-\frac{1}{2}\left(\exp \left(-\frac{1}{2} \operatorname{var}(X+Y)\right)-\exp \left(-\frac{1}{2} \operatorname{var}(X-Y)\right)\right) \\
& =\mathbb{E}\left[\mathrm{e}^{\mathrm{i} X}\right] \mathbb{E}\left[\mathrm{e}^{\mathrm{i} Y}\right] \sinh (\operatorname{cov}(X, Y)),
\end{aligned}
$$

respectively, yielding (3.10).

Indeed, by the random walk representation, if $d \geqslant 3$ then $\operatorname{var}\left(\ell_{1}\left(X_{s}\right)\right)$ and $\operatorname{var}\left(\ell_{2}\left(X_{s+t}\right)\right)$ are uniformly bounded away from 0 , while $\operatorname{cov}\left(\ell_{1}\left(X_{s}\right), \ell_{2}\left(X_{s+t}\right)\right) \rightarrow 0$ whenever $s, t \rightarrow \infty$. Thus, in view of Lemma 3.1 it is clear that no aging takes place. Turning to $d=1,2$, we have that $\operatorname{var}\left(\ell_{1}\left(X_{s}\right)\right) \rightarrow \infty$ and $\operatorname{var}\left(\ell_{2}\left(X_{s+t}\right)\right) \rightarrow \infty$, so it suffices to consider those $s, t \rightarrow \infty$ for which $\operatorname{cov}\left(\ell_{1}\left(X_{s}\right), \ell_{2}\left(X_{s+t}\right)\right) \rightarrow \infty$. By Lemma 3.1 we then have that

$$
\begin{aligned}
\operatorname{corr}\left(f\left(X_{s}\right), g\left(X_{s+t}\right)\right) & \approx \exp \left(\operatorname{cov}\left(\ell_{1}\left(X_{s}\right), \ell_{2}\left(X_{s+t}\right)\right)-\frac{1}{2} \operatorname{var}\left(\ell_{1}\left(X_{s}\right)\right)-\frac{1}{2} \operatorname{var}\left(\ell_{2}\left(X_{s+t}\right)\right)\right) \\
& =\exp \left(-\frac{1}{2} \operatorname{var}\left(\ell_{2}\left(X_{s+t}\right)-\ell_{1}\left(X_{s}\right)\right)\right),
\end{aligned}
$$

and using the Markov property and Gaussian distribution of $\ell_{2}\left(X_{s+t}\right)$ and $\ell_{1}\left(X_{s}\right)$,

$$
\operatorname{var}\left(\ell_{2}\left(X_{s+t}\right)-\ell_{1}\left(X_{s}\right)\right) \geqslant \mathbb{E}\left[\operatorname{var}\left(\ell_{2}\left(X_{s+t}\right)-\ell_{1}\left(X_{s}\right) \mid \mathcal{F}_{s}^{X}\right)\right]=\operatorname{var}\left(\ell_{2}\left(X_{t}\right)\right) \rightarrow \infty,
$$

as $t \rightarrow \infty$, ruling out aging, even in this case.

We conclude with the proof of Proposition 1.3, dealing with the effect that degenerate diffusion coefficients have on aging. 
Proof of Proposition 1.3. (i) In view of (3.2) the term $\operatorname{cov}_{v}\left(P_{s} f, P_{s+t} g\right)$ is negligible, no matter how $t, s \rightarrow \infty$. Turning to deal with the other term in (2.3), recall that $\Gamma(f, g)(x)=a_{0}\left(x_{0}\right) \partial_{0} f(x) \partial_{0} g(x)$, see (2.2). Thus, by the estimate (3.1), for local functions,

$$
\begin{aligned}
\Gamma\left(P_{u} f, P_{u+t} g\right) & \leqslant c_{3}^{2} \sum_{i, j}\left\|\partial_{i} f\right\|_{\infty}\left\|\partial_{j} g\right\|_{\infty} p_{u c_{4}}^{*}(0-i) p_{(u+t) c_{4}}^{*}(0-j) \\
& \leqslant c_{1}(f, g)(u+1)^{-d / 2}(u+t+1)^{-d / 2}
\end{aligned}
$$

Similarly, for monotone local functions $f, g$,

$$
\Gamma\left(P_{u} f, P_{u+t} g\right) \geqslant c_{2}(f, g)(u+1)^{-d / 2}(u+t+1)^{-d / 2} .
$$

Consequently, with $u^{-d}$ integrable for $d \geqslant 2$, we see that no aging takes place then. In case $d=1$ and monotone, local $f, g$, using (2.3) we get that

$$
\operatorname{var}_{v}\left(f\left(X_{s}\right)\right) \approx \log (s+1), \quad \operatorname{var}_{v}\left(g\left(X_{s+t}\right)\right) \approx \log (s+t+1)
$$

and

$$
\begin{aligned}
\operatorname{cov}_{\nu}\left(f\left(X_{S}\right), g\left(X_{s+t}\right)\right) & \approx \int_{1}^{s} u^{-1 / 2}(u+t)^{-1 / 2} \mathrm{~d} u \\
& =2 \log (\sqrt{s}+\sqrt{s+t})-2 \log (1+\sqrt{1+t})
\end{aligned}
$$

which implies (1.11).

(ii) Note that the dynamics for the mean of the super random walk

$$
\mathbb{E} X_{t}(i)=\mathbb{E} X_{0}(i)+\int_{0}^{t}\left(\Delta \mathbb{E} X_{s}\right)(i) \mathrm{d} s,
$$

is linear and translation invariant. Consequently, starting with an initial measure of constant mean, the mean remains constant over time and space:

$$
\mathbb{E}\left[X_{t}(i)\right]=\mathbb{E}\left[X_{0}(i)\right] \equiv m
$$

Since $X_{0} \geqslant 0$, we have that $X$.(.) remains non-negative, and so for monotone functions we get by (3.1) that for some finite $c=c(\alpha)$,

$$
\begin{aligned}
\Gamma\left(P_{u} f, P_{u+t} g\right)\left(X_{s-u}\right) & =\alpha^{2} \sum_{i} X_{s-u}(i)\left(\partial_{i} P_{u} f\right)\left(X_{s-u}\right)\left(\partial_{i} P_{u+t} g\right)\left(X_{s-u}\right) \\
& \leqslant c \sum_{i, j, k} X_{s-u}(i) p_{u c_{4}}^{*}(i-j) p_{(u+t) c_{4}}^{*}(i-k)\left\|\partial_{j} f\right\|_{\infty}\left\|\partial_{k} g\right\|_{\infty} .
\end{aligned}
$$

Consequently, by (3.11), for local monotone functions $f, g$, and some constant $C=C(f, g, m, \alpha)<\infty$, we have that

$$
\mathbb{E}\left[\Gamma\left(P_{u} f, P_{u+t} g\right)\left(X_{s-u}\right)\right] \leqslant C p_{(2 u+t) c_{4}}^{*}(0) \leqslant c(2 u+t+1)^{-d / 2} .
$$

Using (3.3), the same argument produces for $f, g \in \mathcal{C}_{1}^{1, \uparrow}\left(E_{r}\right)$ a lower bound which is comparable up to a universal, finite, positive ratio to the upper bound of (3.12). As the bound (3.2) applies here as well, we conclude by following the computations done in the course of proving Theorem 1.1.

(iii) Similarly to part (ii), the bound (3.2) takes care of the first term in (2.3). We have here too that $\mathbb{E}\left[X_{t}(i)\right]=$ $\mathbb{E}\left[X_{0}(i)\right] \equiv m>0$,

$$
\Gamma(f, g)(x)=\alpha^{2} \sum_{i} x(i)^{2} \partial_{i} f(x) \partial_{i} g(x)
$$

and as the law of $X_{S}(\cdot)$ is invariant under translations, we have that for local monotone functions $f, g$,

$$
\mathbb{E}\left[\Gamma\left(P_{u} f, P_{u+t} g\right)\left(X_{s-u}\right)\right] \approx \mathbb{E}\left[X_{s-u}(0)^{2}\right] p_{(2 u+t) c_{4}}^{*}(0) .
$$


It is well known that

$$
v(s)=\mathbb{E}\left[X_{s}^{2}(0)\right] \approx \begin{cases}\exp (\kappa s+\mathrm{o}(s)), & \alpha>\alpha_{c}, \\ (1+s)^{d / 2-1}, & \alpha=\alpha_{c}, \\ 1, & \alpha<\alpha_{c},\end{cases}
$$

for $\alpha_{c}(d)=\left(\int_{0}^{\infty} p_{2 u}^{*}(0) \mathrm{d} u\right)^{-1 / 2}$ (so in particular, $\alpha_{c}(d)=0$ when $d \leqslant 2$ ), and where $\kappa(\alpha, d)>0$ for any $\alpha>\alpha_{c}$ (cf. [3]). Combining (3.14) and (3.15) it is not hard to verify that no aging applies in this case.

For completeness, we provide here a proof of (3.15), as a simple application of the random walk representation. Indeed note that $\partial_{i} P_{u}(x(0))=\hat{p}_{u}(i, 0)=p_{u}^{*}(i)$ due to (2.7), whereby $P_{u}(x(0))=\sum_{i} x(i) p_{u}^{*}(i)$. Further, from (3.13) we thus get that $\Gamma\left(P_{u}(x(0)), P_{u}(x(0))\right)=\alpha^{2} \sum_{i} x(i)^{2} p_{u}^{*}(i)^{2}$. With $\mathbb{E}\left[X_{s}(i)^{2}\right]=v(s)$ for all $i \in \mathbb{Z}^{d}$ and $s \geqslant 0$, it follows from (2.3) and the independence of $\left\{X_{0}(i)\right\}_{i}$ that

$$
v(s)-m^{2}=\sigma^{2} p_{2 s}^{*}(0)+\alpha^{2} \int_{0}^{s} v(s-u) p_{2 u}^{*}(0) \mathrm{d} u,
$$

where $\sigma^{2}=\operatorname{var}_{v}\left(X_{0}(0)\right)<\infty$. Let $P_{\tau}^{*}(\lambda)=\int_{0}^{\tau} \mathrm{e}^{-\lambda s} p_{2 s}^{*}(0) \mathrm{d} s$. As $P_{\tau}^{*}(\lambda) \uparrow P_{\infty}^{*}(\lambda) \in(0, \infty)$ for $\tau \uparrow \infty$ and any fixed $\lambda>0$, it is easy to check that $V_{\tau}(\lambda)=\int_{0}^{\tau} \mathrm{e}^{-\lambda s} v(s) \mathrm{d} s$ satisfies the inequality

$$
V_{\tau}(\lambda) \leqslant \frac{m^{2}}{\lambda}\left(1-\mathrm{e}^{-\lambda \tau}\right)+\sigma^{2} P_{\tau}^{*}(\lambda)+\alpha^{2} V_{\tau}(\lambda) P_{\tau}^{*}(\lambda) .
$$

We thus conclude that $V_{\infty}(\lambda)<\infty$ as long as $1-\alpha^{2} P_{\infty}^{*}(\lambda)>0$, in which case it is not hard to check directly from (3.16) that

$$
V_{\infty}(\lambda)=\frac{m^{2}+\sigma^{2} \lambda P_{\infty}^{*}(\lambda)}{\lambda\left(1-\alpha^{2} P_{\infty}^{*}(\lambda)\right)}<\infty .
$$

As $\lambda \downarrow 0$ we have that $P_{\infty}^{*}(\lambda) \uparrow P_{\infty}^{*}(0)$ (which is finite for $d \geqslant 3$ and infinite for $d=1,2$ ), so the condition $\alpha>\alpha_{c}=$ $P_{\infty}^{*}(0)^{-1 / 2}$ implies that $\kappa=\inf \left\{\lambda>0: 1-\alpha^{2} P_{\infty}^{*}(\lambda)>0\right\}$ is strictly positive, with $V_{\infty}(\lambda)<\infty$ if and only if $\lambda>\kappa$, hence $v(s)=\exp (\kappa s+\mathrm{o}(s))$. Similarly, when $d \geqslant 3$ and $\alpha<\alpha_{c}$, it follows that $\lambda V_{\infty}(\lambda)$ is bounded as $\lambda \downarrow 0$, and with $v(s)$ differentiable on $(0, \infty)$ (by (3.16) and differentiability of $p_{2 s}^{*}(0)$ ), we get by integration by parts that $v(s) \geqslant m^{2}$ is also bounded above. Analyzing the rate of decay of $\left[P_{\infty}^{*}(0)-P_{\infty}^{*}(\lambda)\right]$ as $\lambda \downarrow 0$, it is easy to resolve also the case $\alpha=\alpha_{c}$.

\section{Aging for dynamics on finite large boxes}

Proof of Theorem 1.4. (i) Assuming fixed boundary conditions, we consider first the case of constant diffusion coefficients (1.5). Then, the random walk representation is of the form

$$
\partial_{i} P_{t} f(x)=\sum_{j} \mathbb{E}_{i, x}\left[\partial_{j} f\left(X_{t}\right) 1_{j}\left(\xi_{t}\right) ; t<\tau_{N}\right]
$$

with $\tau_{N}=\inf \left\{s \geqslant 0: \xi_{s} \notin B_{N}\right\}$ (see [8, formula (1.7)], where the killing at $B_{N}^{c}$ is represented by the usual FeynmanKac term $\exp \left(\int_{0}^{t} u\left(\xi_{s}, X_{s}\right) \mathrm{d} s\right)$, for $\left.u(i, x)=-\sum_{j \notin B_{N}, j-i \in \mathcal{J}} V_{(i, j)}^{\prime \prime}(x(i)-y(j))\right)$. Set

$$
p_{t}^{X, N}(i, j)=\mathbb{E}_{i}\left[1_{j}\left(\xi_{t}\right) ; t<\tau_{N} \mid \mathcal{F}_{t}^{X}\right]
$$

noting that a time reversing argument implies that for all $u \geqslant 0, k, i \in B_{N}$,

$$
p_{u}^{X, N}(i, k)=p_{u}^{\rho_{u}(X), N}(k, i), \quad \text { with } \rho_{u}(X)_{s} \equiv X_{u-s}, 0 \leqslant s \leqslant u .
$$

For two trajectories $X$. and $\widetilde{X}$., and $u>0$ let $\mathbb{P}_{k}^{X, \widetilde{X}, u}$, denote the law of the time inhomogeneous random walk $\left\{\tilde{\xi}_{s}, s \geqslant 0\right\}$ on $\mathbb{Z}^{d}$ starting at $k$ with jump rate

$$
q_{s}(i, j)=V_{(i, j)}^{\prime \prime}\left(X_{u-s}(i)-X_{u-s}(j)\right) 1_{s \leqslant u}+V_{(i, j)}^{\prime \prime}\left(\tilde{X}_{s-u}(i)-\tilde{X}_{s-u}(j)\right) 1_{s>u}
$$


and write

$$
p_{2 u+t}^{X, \widetilde{X}, N, u}(k, j)=\sum_{i \in B_{N}} p_{u}^{X, N}(i, k) p_{u+t}^{\widetilde{X}, N}(i, j)=\mathbb{E}_{k}^{X, \widetilde{X}, u}\left[1_{j}\left(\tilde{\xi}_{2 u+t}\right) ; \tau_{N}>2 u+t\right] .
$$

Next let

$$
\bar{p}_{2 u+t}^{N, u}(k, j)=\sup _{X, \widetilde{X}} p_{2 u+t}^{X, \widetilde{X}, N, u}(k, j), \quad \underline{p}_{2 u+t}^{N, u}(k, j)=\inf _{X, \widetilde{X}} p_{2 u+t}^{X, \widetilde{X}, N, u}(k, j) .
$$

Take $f, g \in \mathcal{C}_{1}^{1, \uparrow}\left(E_{r}\right)$ with $\left|\partial_{k_{0}} f\right|_{\text {inf }}>0$ and $\left|\partial_{j_{0}} g\right|_{\text {inf }}>0$. Since $p_{t}^{X, N}(i, j) \leqslant p_{t}^{X}(i, j)$ of (2.4), the upper bound of (3.2) applies so we shall hereafter neglect the first term of (2.3). Turning to deal with the other term of (2.3), note that by (2.2) and (4.1) we have that for any $u, t \geqslant 0$,

$$
c_{5}(f, g) \underline{p}_{2 u+t}^{N, u}\left(k_{0}, j_{0}\right) \leqslant \Gamma\left(P_{u} f, P_{u+t} g\right) \leqslant c_{6}(f, g) \sup _{j, k} \bar{p}_{2 u+t}^{N, u}(k, j),
$$

taking $k_{0}=j_{0}$ if $f=g$. Clearly,

$$
\bar{p}_{2 u+t}^{N, u}(k, j) \leqslant \bar{p}_{2 u+t}(k, j) \quad \forall u, t \geqslant 0, k, j \in \mathbb{Z}^{d} .
$$

Further, $\inf _{u \leqslant 1} \underline{p}_{2 u}^{N, u}(k, k) \geqslant c_{3}(k)>0$ for any $k$ and all $N$, which implies by (2.3) and the lower bound of (4.3) that for some finite $\overline{s_{0}}$,

$$
\inf _{N} \inf _{s \geqslant s_{0}} \operatorname{var}_{v}\left(f\left(X_{s}\right)\right)>0, \quad \inf _{N} \inf _{s+t \geqslant s_{0}} \operatorname{var}_{v}\left(g\left(X_{s+t}\right)\right)>0 .
$$

Consequently, by the same computations as in proof of Theorem 1.1, it follows that no aging takes place when $d \geqslant 3$.

Turning to deal with $d=1,2$, note that for all $j, k$,

$$
p_{2 u+t}^{X, \widetilde{X}, N, u}(k, j) \leqslant \mathbb{P}_{k}^{X, \widetilde{X}, u}\left(\tau_{N}>2 u+t\right)
$$

and further, for all $j, k$,

$$
p_{2 u+t}^{X, \widetilde{X}, N, u}(k, j) \geqslant p_{2 u+t}^{X, \widetilde{X}, u}(k, j)-\mathbb{P}_{k}^{X, \widetilde{X}, u}\left(\tau_{N}<2 u+t\right) .
$$

We claim that for any $N$ and all $u, t \geqslant 0$,

$$
\sup _{k \in B_{N}} \mathbb{P}_{k}^{X, \widetilde{X}, u}\left(\tau_{N}>2 u+t\right) \leqslant c_{1} \exp \left(-c_{2}(2 u+t) / N^{2}\right),
$$

whereas for any $N$ and all $u, t \geqslant 0$,

$$
\mathbb{P}_{k_{0}}^{X, \widetilde{X}, u}\left(\tau_{N}<2 u+t\right) \leqslant c_{3}(2 u+t+1)\left[\exp \left(-c_{4} N^{2} /(2 u+t)\right)+\exp \left(-c_{5} N\right)\right] .
$$

In view of (4.3) and (4.5), it easily follows from (4.6) and (4.8) that no aging takes place for "small boxes", that is, $N=\mathrm{o}\left((t / \log t)^{1 / 2}\right)$, regardless of the value of $d \geqslant 1$. In contrast, for "large boxes", that is, when $s, t=\mathrm{o}\left(N^{2} / \log N\right)$, the uniform (in $X, \widetilde{X}$ and over $u \leqslant s+t$ ), upper bound of (4.9) decays faster than any fixed power of $s+t$. Thus, by (4.7) and (2.6), it follows that for any $N \geqslant N_{0}$ and all $u \in[0, s+t]$,

$$
2 \underline{p}_{2 u+t}^{N, u}\left(k_{0}, j_{0}\right) \geqslant \underline{p}_{2 u+t}^{u}\left(k_{0}, j_{0}\right) \geqslant c_{1}^{2} p_{(2 u+t) c_{2}}^{*}\left(k_{0}-j_{0}\right) \geqslant c_{5}(2 u+t+1)^{-d / 2} .
$$

Combining (4.4) with the upper bounds of (4.3) and (2.6), we hence conclude that

$$
c_{-}(2 u+t+1)^{-d / 2} \leqslant \Gamma\left(P_{u} f, P_{u+t} g\right) \leqslant c_{+}(2 u+t+1)^{-d / 2},
$$

for positive, finite constants $c_{-}$and $c_{+}$that are independent of $u \leqslant t+s=\mathrm{o}\left(N^{2} / \log N\right)$ and $N \geqslant N_{0}$. This of course implies the same aging statements as in Theorem 1.1.

Turning to prove (4.8), fixing $\Delta>0$, let $\ell=\left\lfloor(2 u+t) / \Delta N^{2}\right\rfloor$, noting that by the Markov property at integer multiples of $\Delta N^{2}$ we have that,

$$
\mathbb{P}_{k}^{X, \tilde{X}, u}\left(\tau_{N}>2 u+t\right) \leqslant \mathbb{P}_{k}^{X, \tilde{X}, u}\left(\tilde{\xi}_{m \Delta N^{2}} \in B_{N}, 1 \leqslant m \leqslant \ell\right) \leqslant\left[\sup _{i \in B_{N}} \sum_{j \in B_{N}} \bar{p}_{\Delta N^{2}}(i, j)\right]^{\ell} .
$$


In view of the upper bounds of (2.5) and (2.6), we can choose $\Delta<\infty$ sufficiently large such that for all $N$ and any $i \in B_{N}$,

$$
\sum_{j \in B_{N}} \bar{p}_{\Delta N^{2}}(i, j) \leqslant \frac{1}{2},
$$

resulting with (4.8).

Moving to deal with (4.9), recall that the random walk $\tilde{\xi}_{s}$ has bounded jump rates $q_{s}(i, j) \leqslant c_{+}$for all $i, j$, and moreover for some $R<\infty$ we have $q_{s}(i, j)=0$ whenever $|j-i|>R$. Consequently, with $Z$ denoting a Poisson $\left(c_{+}\right)$ random variable, it follows that for any $X, \widetilde{X}, m \geqslant 0$ and $u>0$,

$$
\mathbb{P}_{k_{0}}^{X, \tilde{X}, u}\left(\sup _{0 \leqslant \theta \leqslant 1}\left|\tilde{\xi}_{m+\theta}-\tilde{\xi}_{m}\right| \geqslant N / 2\right) \leqslant \mathbb{P}(Z \geqslant N /(2 R)) \leqslant c_{6} \exp \left(-c_{5} N\right),
$$

for some $c_{6}<\infty$ and $c_{5}>0$ depending only on $c_{+}$and $R$. Further, $k_{0} \in B_{N / 4}$ for all $N$ sufficiently large, in which case, the upper bounds in (2.5) and (2.6) imply that for some $c_{4}>0$ and $c_{7}<\infty$, all $u, t \geqslant 0, X$, and $\widetilde{X}$,

$$
\sup _{m \leqslant 2 u+t} \mathbb{P}_{k_{0}}^{X, \widetilde{X}, u}\left(\left|\tilde{\xi}_{m}\right| \geqslant N / 2\right) \leqslant c_{7}\left[\exp \left(-c_{4} N^{2} /(2 u+t)\right)+\exp \left(-c_{5} N\right)\right] .
$$

Combining these two bounds results with (4.9), since

$$
\begin{aligned}
& \mathbb{P}_{k_{0}}^{X, \tilde{X}, u}\left(\tau_{N}<2 u+t\right) \leqslant \mathbb{P}_{k_{0}}^{X, \widetilde{X}, u}\left(\max _{0 \leqslant \theta \leqslant 2 u+t}\left|\tilde{\xi}_{\theta}\right|>N\right) \\
& \quad \leqslant \sum_{m=0}^{\lfloor 2 u+t\rfloor}\left[\mathbb{P}_{k_{0}}^{X, \widetilde{X}, u}\left(\left|\tilde{\xi}_{m}\right| \geqslant N / 2\right)+\mathbb{P}_{k_{0}}^{X, \widetilde{X}, u}\left(\max _{0 \leqslant \theta \leqslant 1}\left|\tilde{\xi}_{m+\theta}-\tilde{\xi}_{m}\right| \geqslant N / 2\right)\right] .
\end{aligned}
$$

In case of linear drift as in (1.3) but non-constant diffusion coefficients we re-run the exact same proof, where by $[8$, formula $(1.7)]$ we then have that

$$
\partial_{i} P_{t} f(x)=\sum_{j} \mathbb{E}_{i, x}\left[\partial_{j} f\left(\widehat{X}_{t}\right) 1_{j}\left(\hat{\xi}_{t}\right) ; t<\hat{\tau}_{N}\right]
$$

for $\hat{\tau}_{N}=\inf \left\{s \geqslant 0: \hat{\xi}_{s} \notin B_{N}\right\}$, instead of (4.1), and throughout

$$
\hat{p}_{t}^{N}(i, j)=\mathbb{P}_{i}\left(\hat{\xi}_{t}=j ; t<\hat{\tau}_{N}\right)=\hat{p}_{t}^{N}(j, i)
$$

replaces $p_{t}^{X, N}(i, j)$ (hence also replacing $\underline{p}_{t}^{N, u}(i, j)$ and $\bar{p}_{t}^{N, u}(i, j)$ ).

(ii) In this case we can also work with the random walk representation, the only change being that the random walk $\tilde{\xi}_{s}$ (or $\hat{\xi}_{s}$, respectively), is restricted to $B_{N}$. Depending on the situation we have periodic jumps or the random walk stays inside $B_{N}$. Thus, the bounds of (4.3) are also valid here, but of course for the corresponding random walk. Let $\operatorname{vol}\left(B_{N}(i, r)\right) \approx(r \wedge N)^{d}$ be the volume of the discrete ball of radius $r$ centered at $i$ within $B_{N}$. Then, for any $k, j \in \mathbb{Z}^{d}$, any $N$ such that $k, j \in B_{N}$, and all $u \geqslant 0, t>0$, we have the estimates

$$
0<\frac{c_{1}(k, j)}{\operatorname{vol}\left(B_{N}\left(k, 1 \vee t^{1 / 2}\right)\right)} \leqslant \mathbb{P}_{k}^{X, \tilde{X}, u}\left(\tilde{\xi}_{t}=j\right) \leqslant \frac{c_{2}}{\operatorname{vol}\left(B_{N}\left(k, 1 \vee t^{1 / 2}\right)\right)}<\infty,
$$

cf. [6]. We thus get from the lower bound of (4.3) that for $d \geqslant 3$ and local monotone functions $f$ and $g$,

$$
\operatorname{var}_{v}\left(f\left(X_{s}\right)\right) \geqslant c_{3} \int_{1}^{s}\left(u^{1 / 2} \wedge N\right)^{-d} \mathrm{~d} u \geqslant c_{4}\left(1+s N^{-d}\right),
$$

and $\operatorname{var}_{v}\left(g\left(X_{s+t}\right)\right) \geqslant c_{4}\left(1+(s+t) N^{-d}\right)$. Combining the obvious bound

$$
\sum_{i}\left\|\partial_{i} P_{s} f\right\|_{\infty}^{2} \leqslant \sum_{j, k}\left\|\partial_{j} f\right\|_{\infty}\left\|\partial_{k} f\right\|_{\infty} \sum_{i \in B_{N}} \sup _{X, \widetilde{X}} \mathbb{P}_{k}^{X, \tilde{X}, s}\left(\tilde{\xi}_{s}=i, \tilde{\xi}_{2 s}=j\right),
$$

with the upper bound of (4.11), which applies also for the above sum over the state $i$ of the random walk at an intermediate time $s$, we get along the same route we took when deriving (3.2), that

$$
\left|\operatorname{cov}_{v}\left(P_{s} f, P_{s+t} g\right)\right| \leqslant c(v, f, g)\left((1+s)^{1 / 2} \wedge N\right)^{-d / 2}\left((1+s+t)^{1 / 2} \wedge N\right)^{-d / 2} .
$$


Since this decays to zero whenever $N, s \rightarrow \infty$ and stays bounded when $N$ does not grow to infinity, we neglect hereafter this term of (2.3), and then get by the upper bounds of (4.3) and (4.11) that

$$
\operatorname{cov}_{v}\left(f\left(X_{s}\right), g\left(X_{s+t}\right)\right) \leqslant c_{5} \int_{1}^{s}\left((2 u+t)^{1 / 2} \wedge N\right)^{-d} \mathrm{~d} u \leqslant c_{6}\left((1+t)^{1-d / 2}+s N^{-d}\right) .
$$

Consequently, for $d \geqslant 3$ and $s=\mathrm{o}\left(N^{d}\right)$ we have that $\operatorname{corr}_{v}\left(f\left(X_{s}\right), g\left(X_{s+t}\right)\right) \rightarrow 0$ as $s, t \rightarrow \infty$.

Keeping with $d \geqslant 3$, when $N=\mathrm{o}\left(s^{1 / d}\right)$ we get from (4.3) and (4.11) matching upper bounds on $\operatorname{var}_{v}\left(f\left(X_{s}\right)\right)$ and $\operatorname{var}_{v}\left(g\left(X_{s+t}\right)\right)$ as well as a matching lower bound on $\operatorname{cov}_{v}\left(f\left(X_{s}\right), g\left(X_{s+t}\right)\right)$. Thus, in this case, $\operatorname{var}_{v}\left(f\left(X_{s}\right)\right) \approx s N^{-d}$, $\operatorname{var}_{v}\left(g\left(X_{s+t}\right)\right) \approx(s+t) N^{-d}$, and $\operatorname{cov}_{v}\left(f\left(X_{S}\right), g\left(X_{s+t}\right)\right) \approx s N^{-d}$, resulting with the stated aging of (1.22).

Proof of Proposition 1.5. Using the notation $(\Delta)(i, j)=\left(\Delta 1_{i}\right)(j)$, the generator of the process $X_{t}(\cdot)$ of $(1.24)$ is

$$
L f(x)=\sum_{i}-(\Delta)^{2}(x)(i) \partial_{i} f(x)+\frac{\alpha^{2}}{2} \sum_{i, j}(-\Delta)(i, j) \partial_{i} \partial_{j} f(x),
$$

and consequently,

$$
\Gamma(f, g)=\alpha^{2} \sum_{i, j} \partial_{i} f(-\Delta)(i, j) \partial_{j} g .
$$

Next let $v_{t}(i, x)=\partial_{i} P_{t} f(x)$, which is the unique solution of

$$
\partial_{t} v_{t}(i, x)=L\left(v_{t}(i, \cdot)\right)(x)-\sum_{j}(\Delta)^{2}(i, j) v_{t}(j, x),
$$

subject to the initial condition $v_{0}(i, x)=\partial_{i} f(x)$, where $(\Delta)^{2}(i, j)=\left(\Delta^{2} 1_{i}\right)(j)=\partial_{i}\left(\Delta^{2}\right)(x)(j)$. Let $A_{t}(i, j)=$ $A_{t}(j, i)=A_{t}(0, j-i)$ for $i, j \in T_{N}$ be the solution of

$$
A_{t}(i, j)=1_{i}(j)-\int_{0}^{t} \sum_{k}(\Delta)^{2}(j, k) A_{s}(i, k) \mathrm{d} s .
$$

Noting that the symmetric matrices $(\Delta)^{2}$ and $A_{t}$ commute, it is not hard to check that

$$
v_{t}(i, x)=\sum_{j} P_{t}\left(\partial_{j} f\right)(x) A_{t}(i, j) .
$$

Thus,

$$
\Gamma\left(P_{u} f, P_{u+t} g\right)=\alpha^{2} \sum_{i, j, \ell, k} P_{u}\left(\partial_{\ell} f\right) A_{u}(\ell, i)(-\Delta)(i, j) P_{u+t}\left(\partial_{k} g\right) A_{u+t}(k, j) .
$$

Considering $f(x)=f(x(0))$ and $g(x)=g(x(0))$ with $0<c_{1} \leqslant f^{\prime}, g^{\prime} \leqslant c_{2}<\infty$, we have that $\kappa=\alpha^{2} P_{u}\left(f^{\prime}\right) P_{u+t}\left(g^{\prime}\right)$ is bounded and bounded away from zero, with

$$
\Gamma\left(P_{u} f, P_{u+t} g\right)=\kappa \sum_{i, j \in T_{N}} A_{u}(0, i)(-\Delta)(i, j) A_{u+t}(0, j) .
$$

To evaluate this convolution sum, recall the Fourier transform

$$
\hat{h}(\theta(k))=\sum_{j \in T_{N}} h(j) \mathrm{e}^{\mathrm{i} \theta(k) \cdot j}
$$

of $h: T_{N} \rightarrow \mathbb{R}$, with $\theta(k)=\frac{2 \pi}{N} k, k \in T_{N}$, which has the inversion formula,

$$
h(j)=\frac{1}{N^{d}} \sum_{k \in T_{N}} \hat{h}(\theta(k)) \mathrm{e}^{-\mathrm{i} \theta(k) \cdot j}
$$


Then, the Fourier transform of $(-\Delta)(0, j)$ is

$$
\hat{a}(\theta(k))=2 \sum_{\ell=1}^{d}\left(1-\cos \left(\theta(k)_{\ell}\right)\right),
$$

and it follows from (4.12) that the Fourier transform of $A_{u}(0, j)$ is

$$
\hat{A}_{u}(\theta(k))=\exp \left(-u \hat{a}(\theta(k))^{2}\right)
$$

resulting with

$$
\Gamma\left(P_{u} f, P_{u+t} g\right)=\frac{\kappa}{N^{d}} \sum_{k \in T_{N}} \hat{A}_{u}(\theta(k)) \hat{a}(\theta(k)) \hat{A}_{u+t}(\theta(k)) .
$$

Recall that $\kappa \in\left[\left(\alpha c_{1}\right)^{2},\left(\alpha c_{2}\right)^{2}\right]$ is bounded and bounded away from zero. Thus, with $\hat{a}(\theta)$ non-negative, so is $\Gamma\left(P_{u} f, P_{u+t} g\right)$. Further, $\hat{a}(\theta) \leqslant 4 d$ for all $\theta$, implying that $\inf _{u \leqslant 1} \Gamma\left(P_{u} f, P_{u} g\right)>0$ and hence both $\operatorname{var}_{v}\left(f\left(X_{s}\right)\right)$ and $\operatorname{var}_{v}\left(g\left(X_{s+t}\right)\right)$ are bounded away from zero uniformly in $s \geqslant 1, t$ and $N$.

By (3.5), we can find $0<c_{-}<c_{+}$such that for any $N$ and all $k \in T_{N}$,

$$
c_{-}(2 \pi)^{2} \frac{|k|^{2}}{N^{2}} \leqslant \hat{a}(\theta(k)) \leqslant c_{+}(2 \pi)^{2} \frac{|k|^{2}}{N^{2}},
$$

implying that for some positive, finite constants $c_{i}$, and all $N, u, t \geqslant 0$,

$$
c_{3} \gamma_{N}\left((2 u+t) c_{4}\right) \leqslant \Gamma\left(P_{u} f, P_{u+t} g\right) \leqslant c_{5} \gamma_{N}\left((2 u+t) c_{6}\right),
$$

where

$$
\gamma_{N}(\tau)=\frac{1}{N^{d}} \sum_{k \in T_{N}} \exp \left(-\tau|k|^{4} N^{-4}\right)|k|^{2} N^{-2} .
$$

Next note that by (1.7), (4.13), Plancherel's identity for the Fourier transform on $T_{N}$ and (4.14), we have that for some $c_{0}(v, f)<\infty, c_{1}>0$ and all $N$,

$$
\begin{aligned}
\operatorname{var}_{v}\left(P_{s} f\right) & \leqslant\left\|C_{v}\right\| \sum_{i}\left\|v_{s}(i, \cdot)\right\|_{\infty}^{2} \leqslant\left\|C_{v}\right\| c_{2}^{2} \sum_{i}\left|A_{s}(i, 0)\right|^{2} \\
& =\frac{\left\|C_{v}\right\| c_{2}^{2}}{N^{d}} \sum_{k \in T_{N}} \hat{A}_{s}(\theta(k))^{2} \leqslant c_{0}(v, f) \eta_{N}(s),
\end{aligned}
$$

where

$$
\eta_{N}(s) \equiv \frac{1}{N^{d}} \sum_{k \in T_{N}} \exp \left(-c_{1} s|k|^{4} N^{-4}\right) .
$$

Turning to bound the covariance of $f\left(X_{S}\right)$ and $g\left(X_{S+t}\right)$, note that $\eta_{N}(s) \leqslant 1$. Hence,

$$
\left|\operatorname{cov}_{v}\left(P_{s} f, P_{s+t} g\right)\right|^{2} \leqslant \operatorname{var}_{v}\left(P_{s} f\right) \operatorname{var}_{v}\left(P_{s+t} g\right) \leqslant c_{2}^{2} \eta_{N}(s+t),
$$

for some $c_{2}=c_{2}(v, f, g)<\infty$.

Consequently, by (2.3), (4.15), and (4.18)

$$
\begin{aligned}
-c_{2} \sqrt{\eta_{N}(t+s)}+c_{3} \int_{0}^{s} \gamma_{N}\left((2 u+t) c_{4}\right) \mathrm{d} u & \leqslant \operatorname{cov}_{\nu}\left(f\left(X_{s}\right), g\left(X_{s+t}\right)\right) \\
& \leqslant c_{5} \int_{0}^{s} \gamma_{N}\left((2 u+t) c_{6}\right) \mathrm{d} u+c_{2} \sqrt{\eta_{N}(t+s)} .
\end{aligned}
$$

Next we claim that as long as $\tau N^{-4}$ is bounded above (and $\tau \geqslant 1$ ),

$$
\gamma_{N}(\tau) \approx \tau^{-(d+2) / 4}, \quad \eta_{N}(\tau) \approx \tau^{-d / 4} .
$$


Indeed, taking $N(\tau)=N / \tau^{1 / 4}$ (which is bounded below), we have that

$$
\begin{aligned}
\tau^{(d+2) / 4} \gamma_{N}(\tau) & =\frac{1}{N(\tau)^{d}} \sum_{k \in T_{N}} \exp \left(-|k / N(\tau)|^{4}\right)|k / N(\tau)|^{2} \\
& \approx \frac{1}{N(\tau)^{d}} \sum_{k \in \mathbb{Z}^{d}} \exp \left(-|k / N(\tau)|^{4}\right)|k / N(\tau)|^{2} \\
& \approx \int_{\mathbb{R}^{d}} \exp \left(-|\theta|^{4}\right)|\theta|^{2} \mathrm{~d} \theta,
\end{aligned}
$$

which is finite and positive. Similarly, then

$$
\tau^{d / 4} \eta_{N}(\tau) \approx \int_{\mathbb{R}^{d}} \exp \left(-|\theta|^{4}\right) \mathrm{d} \theta
$$

verifying (4.20). From the latter and (4.19), it follows that for $d \geqslant 3$ and $(t+s) N^{-4}$ bounded, there exists finite $c_{1}, c_{2}$ such that

$$
\operatorname{cov}_{v}\left(f\left(X_{s}\right), g\left(X_{s+t}\right)\right) \leqslant c_{1}(s+t)^{-d / 8}+c_{2} t^{-(d-2) / 4},
$$

hence no aging. Similarly, for $d=2, t \geqslant 1$ and $(t+s) N^{-4}$ bounded we have

$$
\operatorname{cov}_{v}\left(f\left(X_{s}\right), g\left(X_{s+t}\right)\right) \approx \int_{1}^{s}(2 u+t)^{-1} \mathrm{~d} u \approx \log (2 s+t)-\log (t),
$$

while for $s \geqslant 2$,

$$
\operatorname{var}_{\nu}\left(f\left(X_{s}\right)\right) \approx \int_{1}^{s}(2 u)^{-1} \mathrm{~d} u \approx \log (s), \quad \operatorname{var}_{\nu}\left(g\left(X_{s+t}\right)\right) \approx \int_{1}^{s+t}(2 u)^{-1} \mathrm{~d} u \approx \log (s+t) .
$$

Finally, for $d=1, t \geqslant 1$ and $s \geqslant 1$,

$$
\operatorname{cov}_{v}\left(f\left(X_{s}\right), g\left(X_{s+t}\right)\right) \approx \int_{1}^{s}(2 u+t)^{-3 / 4} \mathrm{~d} u \approx(2 s+t)^{1 / 4}-t^{1 / 4}
$$

with

$$
\begin{aligned}
& \operatorname{var}_{v}\left(f\left(X_{s}\right)\right) \approx \int_{1}^{s}(2 u)^{-3 / 4} \mathrm{~d} u \approx s^{1 / 4}, \\
& \operatorname{var}_{v}\left(g\left(X_{s+t}\right)\right) \approx \int_{1}^{s+t}(2 u)^{-3 / 4} \mathrm{~d} u \approx(s+t)^{1 / 4} .
\end{aligned}
$$

This implies the stated aging results in case $(t+s) N^{-4}$ is bounded. Next consider the small box regime with $N^{4}=$ $\mathrm{o}(s+t)$. Then, by (4.20) and the monotonicity of $\tau \mapsto \eta_{N}(\tau)$, for some $c<\infty$ and any $N \rightarrow \infty$,

$$
\eta_{N}(t+s) \leqslant \frac{c}{N^{d}} \rightarrow 0
$$

regardless of $d \geqslant 1$. Further, in this case, by integration also

$$
\int_{0}^{s} \gamma_{N}((2 u+t) c) \mathrm{d} u \leqslant \int_{0}^{\infty} \gamma_{N}((2 u+t) c) \mathrm{d} u
$$




$$
\begin{aligned}
& \leqslant \frac{c_{3}}{N^{d}} \sum_{k \in T_{N}, k \neq 0} \exp \left(-c t(|k| / N)^{4}\right)(N /|k|)^{2} \\
& \approx \frac{1}{N} \sum_{r=1}^{N} \exp \left(-c t(r / N)^{4}\right)(r / N)^{d-3}
\end{aligned}
$$

which converges to zero if $t \rightarrow \infty$ with $N$ bounded, or even with $N^{4}=\mathrm{o}(t)$ in case $d \geqslant 2$, or $N^{4}(\log N)=\mathrm{o}(t)$ in case $d=1$. Consequently, we have no aging when $N \rightarrow \infty$ such that $N^{4}(\log N)=\mathrm{o}(t)$, if $d=1$ and even $N^{4}=\mathrm{o}(t)$ if $d \geqslant 2$. We complete the proof by showing that for $d \geqslant 3$ there is no aging even when $t N^{-4}$ is bounded while $s N^{-4} \rightarrow \infty$. Indeed, for $\tau \geqslant N^{4}$ we have by (4.16) and (4.20) that

$$
\gamma_{N}(\tau) \leqslant \gamma_{N}\left(N^{4}\right) \exp \left(-\left(\tau-N^{4}\right) N^{-4}\right) \leqslant c_{2} N^{-(d+2)} \exp \left(-\tau N^{-4}\right),
$$

which implies that

$$
\int_{N^{4}}^{\infty} \gamma_{N}(\tau) \mathrm{d} \tau \leqslant c_{3} N^{-(d-2)} \rightarrow 0,
$$

for $N \rightarrow \infty$ and $d \geqslant 3$, thus excluding aging in this case.

\section{References}

[1] G. Ben Arous, Aging and spin-glass dynamics, in: Proceedings of the International Congress of Mathematicians, vol. III, Higher Ed. Press, Beijing, 2002, pp. 3-14.

[2] J.P. Bouchaud, L.F. Cugliandolo, J. Kurchan, M. Mezard, Out of equilibrium dynamics in spin-glasses and other glassy systems, in: A.P. Young (Ed.), Spin Glass Dynamics and Random Fields, World Scientific, 1997.

[3] R. Carmona, S.S. Molchanov, Parabolic Anderson problems and intermittency, Mem. Amer. Math. Soc. 108 (1994).

[4] L.F. Cugliandolo, Dynamics of glassy systems, in: Lecture Notes in Slow Relaxation and Non Equilibrium Dynamics in Condensed Matter, Les Houches, 2002, Session 77.

[5] L.F. Cugliandolo, J. Kurchan, G. Parisi, Off equilibrium dynamics and aging in unfrustrated systems, J. Phys. I (France) 4 (1994) 1691.

[6] T. Delmotte, Parabolic Harnack inequality and estimates of Markov chains on graphs, Rev. Mat. Iberoamericana 15 (1) (1999) $181-232$.

[7] J.-D. Deuschel, Algebraic $L_{2}$-decay of attractive critical processes on the lattice, Ann. Probab. 22 (1994) $264-283$.

[8] J.-D. Deuschel, The random walk representation for interacting diffusion processes, in: J.-D. Deuschel, A. Greven (Eds.), Interacting Stochastic Systems, Springer, Berlin, 2005, pp. 378-391.

[9] T. Delmotte, J.-D. Deuschel, On estimating the derivatives of symmetric diffusions in stationary environment with applications to $\nabla \phi$ interface models, Probab. Theory Related Fields 133 (2005) 358-390.

[10] J.-D. Deuschel, T. Nishikawa, The dynamic of entropic repulsion, Stochastic Process. Appl. (2006), in press.

[11] J.-D. Deuschel, L. Zambotti, Bismut-Elworthy's formula and random walk representation for SDEs with reflection, Stochastic Process. Appl. 115 (2005) 907-925.

[12] J.D. Ferry, Viscoelastic Properties of Polymers, Wiley, New York, 1980.

[13] J. Fröhlich, C.-E. Pfister, On the absence of spontaneous symmetry breaking and of crystalline ordering in two-dimensional systems, Comm. Math. Phys. 81 (1981) 277-298.

[14] T. Funaki, Stochastic interface models, in: Ecole d'été de probabilités de Saint Flour XXXIII - 2003, in: Lecture Notes in Mathematics, vol. 1869, Springer-Verlag, Berlin, 2005, pp. 105-274.

[15] T. Funaki, H. Spohn, Motion by mean curvature from the Ginzburg-Landau $\nabla \phi$ interface models, Comm. Math. Phys. 185 (1997) 1-36.

[16] G. Giacomin, S. Olla, H. Spohn, Equilibrium fluctuations for $\nabla \phi$ interface model, Ann. Probab. 29 (2001) 1138-1172.

[17] A. Greven, Renormalization and universality for multiple population models, in: J.-D. Deuschel, A. Greven (Eds.), Interacting Stochastic Systems, Springer, Berlin, 2005, pp. 209-267.

[18] B. Helffer, J. Sjöstrand, On the correlation for Kac-like models in the convex case, J. Stat. Phys. 74 (1/2) (1994) $349-409$.

[19] M. Mezard, G. Parisi, M. Virasoro, Spin Glass Theory and Beyond, World Scientific Lecture Notes in Physics, 1987.

[20] C.M. Newman, A general central limit theorem for FKG systems, Comm. Math. Phys. 91 (1983) 75-80.

[21] T. Nishikawa, Hydrodynamic limit for the Ginsburg-Landau $\nabla \phi$ interface model with a conservation law, J. Math. Sci. Univ. Tokyo 9 (2002) 481-519.

[22] L.C.E. Struick, Physical Aging in Amorphous Polymers and other Materials, Elsevier, London, 1976. 